\title{
Star Formation and Feedback in Dwarf Galaxies
}

\author{
Shawfeng Dong ${ }^{1}$, D. N. C. Lin ${ }^{1}$ and S. D. Murray ${ }^{2}$
}

\begin{abstract}
We examine the star formation history and stellar feedback effects of dwarf galaxies under the influence of extragalactic ultraviolet radiation. Previous work has indicated that the background UV flux can easily ionize the gas within typical dwarf galaxies, delaying or even preventing cooling and star formation within them. Many dwarf galaxies within the Local Group are, however, observed to contain multiple generations of stars, the oldest of which formed in the early epochs of cosmic evolution, when the background UV flux was intense. In order to address this paradox, we consider the dynamical evolution of gas in dwarf galaxies using a one-dimensional, spherically symmetric, Lagrangian numerical scheme to compute the effects of radiative transfer and photoionization. We include a physically-motivated star formation recipe and consider the effects of feedback. This scheme allows us to follow the history of the gas and of star formation within dwarf galaxies, as influenced by both external and internal UV radiation. Our results indicate that star formation in the severe environment of dwarf galaxies is a difficult and inefficient process. In potentials with total mass less than a few $10^{6} M_{\odot}$, and velocity dispersion less than a few $\mathrm{km} \mathrm{s}^{-1}$, residual gas is efficiently photoionized by cosmic background UV radiation. Since the density scale height of the gas within these galaxies is comparable to their size, gas may be tidally removed from them, leaving behind starless residual dark matter clumps. For intermediate mass systems, such as the dSphs around the Galaxy, star formation can proceed with in early cosmic epochs despite the intense background UV flux. Triggering processes such as merger events, collisions, and tidal disturbance can lead to density enhancements, reducing the recombination timescale, allowing gas to cool and star formation to proceed. However, the star formation and gas retention efficiency may vary widely in galaxies with similar dark matter potentials, because they depend on many factors, such as the baryonic fraction, external perturbation, IMF, and background UV intensity. We suggest that the presence of very old stars in these dwarf galaxies indicates that their initial baryonic to dark matter content was comparable to the cosmic value. This constraint suggests that the initial density fluctuation of baryonic matter may be correlated with that of the dark matter. For the more massive dwarf elliptical galaxies, the star formation efficiency and gas retention rate is much higher. Their mass to light ratio is regulated by star formation feedback, and is expected to be nearly independent of their absolute luminosity. The results of our theoretical models reproduce the observed $M / L-M_{v}$ correlation.
\end{abstract}

Subject headings: galaxies: dwarf — galaxies: formation — stars: formation — radiative transfer

\footnotetext{
${ }^{1} \mathrm{UCO} /$ Lick Observatory, University of California, Santa Cruz, CA, 95064

Email: dong@ucolick.org, lin@ucolick.org

${ }^{2}$ University of California, Lawrence Livermore National Laboratory, P.O. Box 808, Livermore, CA 94550 Email: sdmurray@llnl.gov
} 


\section{Introduction}

According to the popular cosmological models dominated by cold dark matter (CDM), the formation of large galaxies occurs in a hierarchical fashion, through the coalescence of smaller systems (Blumenthal et al. 1984; Navarro, Frenk, \& White 1997; Klypin, Nolthenius, \& Primack 1997). During mergers, the dissipationless dark matter components combine to form the extended dark halos of larger galaxies. The gas of the dwarf systems, however, dissipates its kinetic energy during mergers, decouples from the dark matter, and falls either into the center or onto the equatorial plane, forming the bulge and disk. Dwarf galaxies observed today, such as the dwarf spheroidal (dSph) satellites of the Milky Way, represent surviving members of the original population of building blocks from which the Milky Way was formed.

Along with the success of this picture, however, has come the realization of new problems, both in the theory and in comparisons with observations. Three problems are of particular interest here. One difficulty is the so-called "over-cooling problem." In the CDM scenario, the magnitude of linear density fluctuations, $\sigma(M) \equiv\left\langle(\delta M / M)^{2}\right\rangle^{1 / 2}$ is a decreasing function of the mass scale, $M$. Such a fluctuation spectrum causes low mass systems to become nonlinear and virialize at earlier epochs, and within a more dense background, than larger systems. Cooling is very efficient at early epochs, due both to the generally higher densities at high red redshift as well as the rapid increase in the rate of inverse Compton cooling with redshift. The early growth of smaller systems, coupled with the more efficient cooling at early epochs, leads to the expectation of rapid condensation of small, dense gas clouds within the dark-matter halos of dwarf galaxies. But, unless star formation can be suppressed, most of the gaseous ordinary matter would be converted into stars well before these galactic building blocks can be assembled into present-day large galaxies such as the Milky Way (White \& Rees 1978).

A second problem is the deficit of observed satellite dwarf galaxies relative to theoretical expectations. Observationally, the CDM scenario naturally leads to a "bottom-up" hierarchical galaxy formation picture that is qualitatively consistent with dwarf galaxies having halos with higher phase-space densities than those around larger normal galaxies. High-resolution numerical simulations show, however, that dwarf galaxies with higher internal densities retain the integrity of their cores while their loosely bound halos merge with each other during the formation of larger galaxies (Moore et al. 1999; Klypin et al. 1999). Consequently, the number density of surviving satellite dwarf galaxies expected from the CDM scenario is much larger than that observed in the Local Group. Early bursts of star formation could lead to the preservation of ordinary matter in a gaseous form, followed by its removal from the dark matter potential. Such a process may account for the deficit of detectable satellite galaxies.

The third problem involves the building up of large galaxies from the dwarf systems. Under the action of dynamical friction from the surrounding sea of dark matter, the dwarf galaxy building blocks lose energy and orbital angular momentum before they eventually merge into larger disk galaxies. If the gas within the dwarf systems cools and contracts within their dark matter potentials, the mergers are delayed, leading to the formation of disk systems which are much smaller and denser than observed (e.g., Navarro \& Benz 1991; Navarro \& White 1994). The separation of gas from the dark matter potential of dwarf galaxies is one possible mechanism for the ordinary matter to retain sufficient angular momentum to form normal-size disk galaxies (Weil, Eke \& Efstathiou 1998; Navarro \& Steinmetz 1997; Sommer-Larsen, Gelato \& Vedel 1999).

Resolution of the above problems may require that the conversion of gas into stars within the dwarf systems be inefficient. Recent theoretical investigations suggest that the conversion of gas into stars in dwarf galaxies may be suppressed by the background UV radiation at large redshifts (Weinberg, Hernquist \& Katz 1997; Kepner, Babul \& Spergel 1997). Integrating the full equations of radiative transfer, heating, cooling and 
non-equilibrium chemistry for nine species: $\mathrm{H}, \mathrm{H}, \mathrm{H}^{+}, \mathrm{H}^{-}, \mathrm{H}_{2}, \mathrm{H}_{2}^{+}, \mathrm{He}, \mathrm{He}^{+}, \mathrm{He}^{++}, \mathrm{e}^{-}$, Kepner et al. (1997) computed the quasi-hydrostatic equilibrium states of gas in spherically symmetric dark matter potentials roughly corresponding to those of dwarf galaxies, and found that a typical background UV radiation field can easily delay cooling and collapse of gas in halos corresponding to $1 \sigma \mathrm{CDM}$ perturbations with circular velocities less than $30 \mathrm{~km} \mathrm{~s}^{-1}$.

Those results, however, introduce an inconsistency with other observed properties of low-mass dwarf galaxies. The color-magnitude diagrams of most dSphs indicate that star formation began within them at an early epoch. Indeed, Ursa Minor and Draco, whose stellar velocity dispersions are only a few $\mathrm{km} \mathrm{s}^{-1}$, and whose total masses are less than $10^{6} M_{\odot}$, are composed primarily of very old stars.

In some dSphs, such as Carina and Fornax, the coexistence of multiple generations of young and intermediate-age stars (Grebel 1997; Grebel \& Stetson 1998) provides evidence of protracted and recent star formation episodes. This is again difficult to account for, because little atomic or molecular gas is found in them today (Knapp, Kerr \& Bowers 1978; Mould et al. 1990; Bowen et al 1997; Carignan et al. 1998), though some gas has been detected around some more distant dSphs, such as Sculptor. While it is natural to expect that either internal star formation or external ram-pressure stripping might easily remove any residual gas from the shallow potentials of dSphs (Noriega-Crespo et al. 1989; Mori \& Burkert 2000), it is difficult to reconcile the lack of either cool or warm gas with the presence of young stars.

A more complete picture of the early evolution of dwarf galaxies, and of their role in the formation of massive systems, therefore requires a more thorough understanding of the various physical processes, both external and internal, that act to trigger and regulate star formation within them. In this paper, we present an investigation of the hydrodynamic evolution and star formation history of dwarf galaxies. We construct a code which integrates a realistic star formation recipe, feedback, and radiative transfer in a 1D spherically symmetric Lagrangian scheme. The code allows us to follow the evolution of both the state of the gas and the star formation of dwarf galaxies subjected to different background UV fluxes and different external perturbations. In examining the parameter space, we seek answers to the following questions:

1). Is star-formation in dwarf galaxies triggered by a decrease in the background UV radiation, or by external perturbations?

2). What is the star formation efficiency, i.e. the fraction of the initial mass of gas that is converted into stars?

3). What is the mechanism for triggering multiple bursts of star-formation, such as are observed in some dSphs?

In $\S 2$ of this paper, we describe the dwarf galaxy model, the star formation recipe, feedback, and the Strömgren shell model for radiative transfer and photoionization. We combine all of these into a 1D spherically symmetric Lagrangian hydrodynamical scheme. In $\S 3$ we discuss the results of our simulations, and in $\S 4$ we discuss the implications of our results.

\section{Numerical Models}

A wide range of physical processes and physical scales are associated with galaxy and star formation. Including all of them would be a formidable task, beyond the capabilities of even the most powerful current generation of computers. In this work, we simplify the problem by examining the time evolution of a 
spherically symmetric cloud, and combine models for the most important processes, including radiative transfer, photoionization, star formation, and feedback. The problem therefore becomes tractable, and the results which we obtain may be included in larger-scale simulations.

\subsection{Dark Matter Halo}

We simulate the evolution of the gas within a fixed halo potential. Similar to the approach adopted by Kepner et al. (1997), we choose a form for the potential found by Burkert (1995), which has been shown to be a good fit to many dwarf systems. The corresponding dark matter density distribution is given by

$$
\rho_{D M}=\frac{\rho_{0} r_{0}^{3}}{\left(r+r_{0}\right)\left(r^{2}+r_{0}^{2}\right)}
$$

where

$$
\rho_{0}=4.5 \times 10^{-2}\left(r_{0} / \mathrm{kpc}\right)^{-2 / 3} \mathrm{M}_{\odot} \mathrm{pc}^{-3}
$$

is the central density. The dark matter density tends to $\rho \propto$ const. for $r \ll r_{0}$, and $\rho \propto r^{-3}$ for $r \gg r_{0}$. The rotational velocity, and mass at $r_{0}$ are given by

$$
\begin{aligned}
v_{0} & =17.7\left(r_{0} / \mathrm{kpc}\right)^{2 / 3} \mathrm{~km} \mathrm{~s}^{-1} \\
M_{0} & =7.2 \times 10^{7}\left(r_{0} / \mathrm{kpc}\right)^{7 / 3} \mathrm{M}_{\odot}
\end{aligned}
$$

In the numerical simulations presented below, the above mass distribution is arbitrarily assumed to extend out to a halo radius

$$
r_{\text {halo }}=3.4 r_{0}
$$

and so the total mass of the dark matter halo is $M_{D M}=4 \pi \int_{0}^{r_{h a l o}} \rho_{D M} r^{2} d r=5.8 M_{0}$.

\subsection{Gaseous Component}

The dark matter halo contains ordinary matter in the forms of stars and gas. The evolution of the gas density, $\rho_{g}$ is described by the continuity equation,

$$
\frac{\mathrm{d} \rho_{g}}{\mathrm{~d} t}+\rho_{g} \nabla \cdot \mathbf{v}=-\dot{\rho_{s}}
$$

The sink term $\dot{\rho}_{s}$, due to star formation, denotes the rate by which the gas becomes gravitationally unstable (see Equation 2-11). In the above expression, $\mathrm{d} / \mathrm{d} t$ is the material derivative and $\mathbf{v}$ is the gas velocity.

The momentum equation for the gas is

$$
\rho_{g} \frac{\mathrm{d} \mathbf{v}}{\mathrm{d} t}=\rho_{g} \mathbf{g}+\nabla p+\nabla p_{k}
$$

where the force $\mathbf{g}=-\nabla \psi$ is due to the gradient of the gravitational potential $\psi, p$ is the gas pressure, and we introduce a kinetic pressure, $p_{k}$ to represent the momentum input due to stellar wind and supernovae of massive stars (see Equation 2-15).

Massive stars emit intense UV radiation, providing another mechanism for feedback. In addition, observations of Ly $\alpha$ clouds along quasar lines-of-sight suggest that, at high redshifts, the universe is permeated by a metagalactic UV flux. At $z=2$, this background is estimated to have a strength $J_{\nu} \approx$ 
$10^{-21} \mathrm{ergs} \mathrm{s}^{-1} \mathrm{~cm}^{-2} \mathrm{ster}^{-1} \mathrm{~Hz}^{-1}$ at the Lyman limit (Bechtold et al 1987). Both of these energy sources are modelled in the energy equation of the gas,

$$
\frac{\mathrm{d} \epsilon}{\mathrm{d} t}+p \frac{\mathrm{d} V}{\mathrm{dt}}=G-L,
$$

where $\epsilon$ is the specific internal energy, $V=1 / \rho_{g}$, and $G$ and $L$ represent radiative heating and cooling terms.

\subsection{Prescription for Star Formation and Feedback}

In order to consider the effect of feedback, the formation rate of massive stars must be specified. Here, we introduce a simple prescription for massive star formation. In general, gas is destabilized by its collective self-gravity and stablized by internal pressure and external tidal stress. When the residual gas within a galaxy is fully ionized, it attains hydrostatic equilibrium, with the Jeans mass $M_{J}$ of the system comparable to or larger than the total mass of gas. But, if the gas is able to cool well below the ionization temperature, $M_{J}$ decreases to masses comparable to high mass stars. The density of the residual gas also increases as it contracts to a new hydrostatic equilibrium within the dark matter potential. In those regions of the dwarf galaxy where $\rho_{g}>3\left\langle\rho_{D M}(r)\right\rangle$, with

$$
\left\langle\rho_{D M}(r)\right\rangle=\left(3 / r^{3}\right) \int_{0}^{r} \rho_{D M}\left(r^{\prime}\right) r^{\prime 2} d r^{\prime},
$$

the tidal field of the potential is insufficient to prevent the collapse of gas within the Jeans radius $R_{J}=$ $\sqrt{\frac{3 R_{g} T}{1.6 \pi G \rho_{g} \mu}}$. In the absence of a strong magnetic field, the gas collapses to form stellar mass objects when these conditions are satisfied.

Ionizing photons and stellar winds from young, massive stars act to raise the Jeans mass of surrounding gas, inhibiting further star formation. The collapse of the gas cloud does not, however, lead to immediate star formation and feedback. A minimum time delay between the instant when the gas becomes gravitationally unstable and the onset of main sequence evolution for the resulting generation of stars is the dynamical free-fall time scale $\tau_{f f}=\left(G \rho_{g}\right)^{-1 / 2}$. If the collapsing gas has angular momentum, protostellar disks would form, through which gas is accreted onto the growing protostellar objects on a viscous time scale. After the formation of the protostars, an additional time interval, $\tau_{\text {onset }}$, must elapse before the emission of intense UV radiation. For most of our models, we choose $\tau_{\text {onset }}=0.5 \mathrm{Myr}$. But in some delayed star formation models, we choose $\tau_{\text {onset }}=$ either 5 or $50 \mathrm{Myr}$, to represent delays due to disk accretion (Yorke \& Sonnhalter 2002), merger processes (Murray \& Lin 1996, Bonnel et al. 1998), or sequential star formation (Elmegreen $\&$ Lada 1977). Thus, changes in the stellar and gas mass densities $\left(\rho_{\star}\right.$ and $\rho_{g}$ respectively) are related via a time-delay, such that

$$
\dot{\rho}_{\star}(t)=\dot{\rho}_{s}\left(t-\tau_{f f}-\tau_{\text {onset }}\right)
$$

and,

$$
\dot{\rho_{s}}=\beta \frac{\rho_{g}}{\tau_{f f}}
$$

where $\tau_{f f}$ refers to the local free-fall timescale at the epoch when the collapse begins, i.e. $t-\tau_{f f}-\tau_{\text {onset }}$. We adopt a form for the star formation efficiency, $\beta$, of

$$
\beta=\left(\frac{\tau_{\text {onset }}}{\tau_{\text {onset }}+\tau_{f f}}\right) \frac{\beta_{\max }}{\left[1+\left(\frac{T}{T_{J}}\right)^{\lambda_{T}}\right]\left[1+\left(\frac{R_{J}}{R_{R}}\right)^{\lambda_{R}}\right]}
$$


where $T_{J}=0.4\left(\frac{4 \pi}{3}\right)^{\frac{1}{3}} \mu G M_{s}^{\frac{2}{3}} \rho_{g}^{\frac{1}{3}}$ is the critical temperature for the onset of $M_{s}=100 M_{\odot}$ star formation, and $R_{R}$ is the Roche radius of the gas in the potential of the dwarf galaxy. The three factors in $\beta$ act to decrease the star formation efficiency in situations where it is expected to be low. When the local free fall time is longer than the timescale for the onset of main sequence evolution, then it would be expected that, because of the spread in star formation times, the first stars to form would inhibit further star formation. The efficiency is reduced for high stellar masses, due to the fact that the dynamical timescale for the evolution of the gas exceeds the evolution time of massive stars, in which case the first stars to form would re-ionize the surrounding gas before it, too, could collapse to form stars. Finally, star formation is also expected to be inefficient whenever the Roche radius of a Jeans unstable region exceeds its tidal radius within the dwarf galaxy. We set the star formation quenching factors $\lambda_{T}=\lambda_{R}=4$, such that star formation is rapidly shut off whenever one or more of the above criteria is met. Unless otherwise stated, we set the maximum efficiency $\beta_{\max }=1$. In some cases, we choose $\beta_{\max }=0.1$, representing a conversion of at most ten percent of the total residual gas into stars within a free fall time.

Accompanying each massive progenitor star, there are many coeval lower-mass stars. Thus, the corresponding formation rate, per unit volume, of massive UV and wind producing stars is given by

$$
\dot{n_{\star}}=\frac{\dot{\rho_{\star}}}{M_{c}} .
$$

The total mass of stars formed per UV, wind, and supernova producing star, $M_{c}$, can be estimated from a Scalo initial mass function (IMF) to be $150 M_{\odot}$ (Scalo 1986; Miller \& Scalo 1979). For more general initial mass functions, we set

$$
M_{c}=f_{I M F} 150 M_{\odot},
$$

where $f_{I M F}>1$ for a steeper initial mass function with a relatively small cut off in the stellar mass function at the high end. Unless otherwise stated, $f_{I M F}=1$ is chosen for most of our models. In order to explore the effects of variations, we examine some models in which $f_{I M F} \gg 1$.

The formation of massive stars strongly influences the environment. The massive stars emit UV radiation with a well-determined flux (see e.g. Osterbrock 1989). When averaged over a Miller-Scalo initial mass function, the rate of ionizing photon emission per $10 M_{\odot}$ star is $\bar{Q}_{\star}=10^{48.5} \mathrm{~s}^{-1}$. Photoionization by UV radiation from massive stars can be included in the energy equation of the gas, and in determining its ionization state (see below). Stars are assumed to emit UV photons at an average rate of $\bar{Q}_{\star}$ for a time equal to the average lifetime of a massive star, $\tau_{\star}=4$ Myr. After that time, they are taken to be "dead stars," contributing to the overall mass of the system, but providing no feedback to the gas.

After a brief stage of adiabatic expansion away from the massive stars, the main effect of the stellar winds and supernovae is to increase the momentum of the surrounding gas. We approximate this feedback process as a a "kinetic pressure" term in Equation (2-7), defined for each given volume (zone) of the model as

$$
p_{k}=\frac{d \dot{N}_{s n}}{d S} P_{s n}+\frac{d N_{\star}}{d S} \dot{m}_{w} v_{w},
$$

where the surface area at the center of the zone is $S$. The first term in Equation 2-15 is the momentum flux due to supernovae, and the second is that due to stellar winds. In the above equation, $P_{s n}$ is the momentum of a single supernova explosion (we adopt $4.5 \times 10^{42} \mathrm{~g} \mathrm{~cm} \mathrm{~s}^{-1}$ ), $N_{s n}$ is the total supernova rate (number of SN per unit time) within the volume of the zone. The value of $\dot{N}_{s n}$ is obtained from the the supernova rate per unit volume, which itself is determined by the star formation rate at the earlier time $t-\tau_{\star}$, i.e.

$$
\dot{n}_{s n}(t)=\dot{n}_{\star}\left(t-\tau_{\star}\right) .
$$


The total number of massive stars on the main sequence within the volume of the zone is $N_{\star}$, while the wind mass loss rate of a typical massive star and the typical wind speed are $\dot{m}_{w}\left(=10^{-6} M_{\odot} \mathrm{y}^{-1}\right)$ and $v_{m}\left(=10^{3}\right.$ $\mathrm{km} \mathrm{s}^{-1}$ ), respectively.

With these prescriptions for the feedback process, we use a one-dimensional Lagrangian hydrodynamic model for the evolution of the baryonic gas component within the fixed external gravitational potential set by the dark matter (Richtmyer \& Morton 1995).

\subsection{Photoionization and Radiative Transfer}

As indicated above, UV radiation from both massive stars and the background can lead to photoionization and heating of the residual gas. A comprehensive treatment of the full $3 \mathrm{D}$ radiative transfer requires the determination of the contribution to the flux at every point from every other point along all paths for each wavelength, which is at the minimum a $6 \mathrm{D}$ problem. However, in most instances, symmetries can be introduced, resulting in a more tractable situation. The greatest simplification occurs when the gas can be assumed to be optically thin throughout. This approximation is sufficient in the majority of cosmological situations (Katz, Weinberg \& Hernquist 1996; Navarro \& Steinmetz 1996; Anninos, Norman \& Clarke 1994) and only breaks down in the cores of halos that have undergone sufficient cooling, a situation that is usually made intractable by the complexities of star formation.

The next simplest geometry is that of a slab (or a sphere under the assumption of a radially perpendicular radiation field), which reduces the flow to an intrinsically $2 \mathrm{D}$ problem. This approach is the most common in radiative transfer, and has been used to address similar situations to those considered here (Hainman, Rees \& Loeb 1996; Haiman, Thoul, \& Loeb 1996).

In this paper, we adopt a similar approach for spherical geometry. We relax the assumption of a radially perpendicular radiation field by treating radiative transfer using the Strömgren shell model. Similar to the original Strömgrem model, the ionized " $\mathrm{H}$ II" shell is assumed to be surrounded isotropically by a neutral $\mathrm{H}^{0}$ region (Figure 1). The rate of entry of ionizing photons into the ionized volume balances the total number of recombinations to excited levels within the volume.

In our implementation of the Strömgrem shell model, we use a discretized 1D Lagrangian hydrodynamic scheme. We define

$$
Q_{j}=V_{j} n_{H}^{2} \alpha_{B}
$$

to be the number of photons required to ionize zone $j$, with $V_{j}$ being the volume of the zone, $n_{H}$ being the number density of hydrogen, and $\alpha_{B}$ being the case B recombination coefficient (Osterbrock 1989). The total number of UV photons emitted by the massive stars in the zone is $F_{\star, j}$, while $F_{i n, j}$ is the rate of UV photons entering into the zone from larger radii, and $F_{\text {out }, j}$ is the rate of UV photons entering into the zone from smaller radii (Figure 2 ). In the innermost zone, there is only $F_{\star}$ and $F_{i n}$.

The total rate of ionizing photons either produced within or entering into the zone is $F_{t, j}=F_{\star, j}+$ $F_{i n, j}+F_{o u t, j}$. If $F_{t, j}<Q_{j}$, then the zone is only partially ionized, and the ionization fraction of the gas is given by

$$
X_{j}=F_{t, j} / Q_{j} .
$$

If $F_{t, j}>Q_{j}$, then $X_{j}=1$, and any photons in excess of $Q_{j}$ give rise to $F_{o u t, j+1}$ and $F_{i n, j-1}$ of adjacent 
zones, as follows;

$$
F_{i n, j-1}=f_{g} \times\left(\frac{1}{2} F_{\star, j}+F_{i n, j}-\frac{1}{2} Q_{j}\right)
$$

and

$$
F_{\text {out }, j+1}=\frac{1}{2} F_{\star, j}+F_{\text {out }, j}-\frac{1}{2} Q_{j}+\left(1-f_{g}\right) \times\left(\frac{1}{2} F_{\star, j}+F_{\text {in }, j}-\frac{1}{2} Q_{j}\right) .
$$

The term

$$
f_{g}=\left(r_{\text {in }} / r_{\text {out }}\right)^{2}
$$

with $r_{\text {in }}$ and $r_{\text {out }}$ being, respectively, the inner and outer radii of any zone, is a factor that takes into consideration the spherical geometry of the model. In the limit of isotropic radiation, that geometry causes slightly more than half of the photons within a zone to exit in the outward direction. The case of either slab geometry or of a radially-directed radiation field can be represented by $f_{g}=1$. The above form of $f_{g}$ can be arrived at by considering the optically thin limit with only inflowing UV photons. In that case, the UV intensity $J_{\nu}\left(r_{\text {in }}\right) \approx J_{\nu}\left(r_{\text {out }}\right)$, and the flux of photons $F \propto r^{2}$, thus $F\left(r_{\text {in }}\right) / F\left(r_{\text {out }}\right) \approx\left(r_{\text {in }} / r_{\text {out }}\right)^{2}$. Care is also taken to deal with the special cases when either $F_{i n, j-1}$ or $F_{o u t, j+1}$ is negative. Iterations by sweeping inwards and then outwards several times are taken to ensure convergence. For the innermost zone, $F_{\text {out }}=0$, while for the outermost zone $F_{\text {in }}$ is given by the external radiation field.

The external UV flux $J_{\nu} \approx 10^{-23} \mathrm{ergs} \mathrm{s}^{-1} \mathrm{~cm}^{-2} \mathrm{ster}^{-1} \mathrm{~Hz}^{-1}$ at the Lyman limit at low red shift, and 50 times more intense at redshift $z=3$ (cf. Haardt \& Madau 1996), is sufficient to ionize most dwarf spheroidal galaxies throughout. For a less intense external flux, neutral residual gas is surrounded by ionized gas. The presence of metals would increase the critical $J_{\nu}$ needed for fully ionizing the dwarf galaxy, but, because we are considering the early evolution of low-metallicity systems, the modification is expected to be limited. The temperature of the photoionized gas is taken to be $15,000 \mathrm{~K}$, appropriate for low-metallicities. We calculate the temperature for the $\mathrm{H}^{0}$ region according to Equation 2-8. We adopt equilibrium cooling rates, including contributions from hydrogen, helium, and metals (Dalgarno \& McCray 1972; Fall \& Rees 1985). Because the dwarf galaxies being modeled have low metallicities, and we are modeling them at early epochs, the metallicity is taken to be low, $[\mathrm{Fe} / \mathrm{H}]=-2$. In the absence of photoionization, such metal-poor gas gas may cool to a minimum temperature of $\sim 10^{2} \mathrm{~K}$ (Kang et al. 1990; Lin \& Murray 1992). Using the above treatments for heating, cooling, and radiative transfer, we can compute the ionization fraction and the temperature of the gas as well as the attenuation of the ionizing photons.

We note that the model described above follows the assumptions used in calculating static, "initial" Strömgren spheres. These are formed extremely rapidly, on timescales of the order of the recombination time of the gas $\left(\sim 10^{8} \mathrm{~s}\right)$. Subsequently, the gas expands in response to the photoionization heating. To compute, on galactic scales, the evolution of individual Strömgren spheres, as well as that of the ionization front propagating inwards from the external UV radiation, is a multidimensional problem, beyond current computational capabilities. Our models do capture the expansion of the ionized gas within each zone in response to heating, and therefore account for its global effects upon feedback and star formation. The essential physics of the problem is therefore included in the models.

Our models also assume the gas to be uniformly distributed within each zone. Clumpiness in the ISM would increase the value of $Q_{j}$ from that given above. Clumpiness would result from either temperature variations (a multi-phase medium) or self-gravity. In the former case, the presence of a cold medium implies the lack of sufficient UV photons to completely ionize the galaxy, and that subsequent star formation shall rapidly lead to re-heating and ionization of the gas, ie. in our models, this corresponds to a dwarf galaxy 
undergoing star formation. If the gas is completely heated, the Jeans mass is sufficiently large that self-gravity shall be unable to cause significant clumping.

Finally, our models do not include metallicity evolution of the gas, due to star formation. Metallicity does not strongly affect the size of Strömgren spheres, and so this shall not significantly affect our assumptions regarding ionization by external and internal UV sources. The primary affect of increasing metallicity is to enhance cooling in gas that has lost its heat source, potentially enhancing the star formation efficiency. This, too, is not likely to be a strong effect, because the timescales for star formation are dominated by the initial free-fall time of the gas, and the pre-main sequence evolution time of the stars. The main affect would, therefore, likely be in the stellar mass function. Higher metallicity gas can cool to lower temperatures, possibly enhancing the formation of low mass stars, and reducing the effectiveness of internal feedback. Such effects must be included in the uncertainties of our models, and we explore a wide range of parameters in order to examine their potential significance.

\section{Dwarf Galaxy Models}

\subsection{Model Parameters}

The currently preferred (e.g. de Bernardis et al 2002) cosmological parameters are as follows: the Hubble constant is

$$
H_{0}=100 h \mathrm{~km} \mathrm{~s}^{-1} \mathrm{Mpc}^{-1}=71 \pm 3.5 \mathrm{~km} \mathrm{~s}^{-1} \mathrm{Mpc} ;
$$

the age of the universe is $t_{0}=13.7 \pm 0.1 \mathrm{Gyr}$; the baryonic density parameter is $\Omega_{b}=0.04$; and the dark matter density parameter is $\Omega_{m}=0.23$.

These parameters are relevant to the issues of delayed star formation in dwarf galaxies. For instance, Kepner et al. (1997) simulated halos in the range of $15 \mathrm{~km} \mathrm{~s}^{-1}<\mathrm{v}_{\mathrm{c}}<70 \mathrm{~km} \mathrm{~s}^{-1}$, which, using their model, translate to: $3 \times 10^{8} M_{\odot}<M_{\text {halo }}<5 \times 10^{10} M_{\odot}$. In their models, $3 \times 10^{5} M_{\odot}<M_{\text {gas }}<5 \times 10^{9} M_{\odot}$, such that the baryonic fraction is:

$$
10^{-3}<b \equiv M_{\text {gas }} / M_{\text {halo }}<0.1 .
$$

With the very small value of $b=0.001$, dwarf galaxies at the low end of the mass range could not form stars even today because the recombination efficiency is insufficient to offset photoionization due to external background UV radiation. Kepner et al. (1997) deduced such a small value for $b$ under the assumption that the ordinary matter is accreted onto the dark matter halo from an ionized intergalactic medium with temperature $T=2 \times 10^{4} \mathrm{~K}$. However, if the dwarf galaxies formed prior to the era of reionization or if the first generation stars are primarily formed in the progenitors of dwarf galaxies, then cool, neutral gas may be retained by their dark matter haloes with the cosmological value of $b=\Omega_{b} / \Omega_{m} \sim 0.17$.

Observation of dwarf galaxies in the local group find high baryonic fractions (Mateo 1998). Indeed, in some systems, such as NGC 147, the observationally inferred estimate of $b$ approaches unity. Such values indicate that these systems may have undergone complicated and highly varied evolutionary histories. It is possible that dwarf galaxies had larger values of $b$ in the past, and have since preferentially lost a fraction of their baryonic matter through star formation feedback (Dekel \& Silk 1986) and tidal stripping (Murray, Dong, \& Lin 2003). It is also possible that the observed dwarf galaxies are completely different from the dwarf galaxy building blocks in the CDM scenario. In view of these uncertainties, it is useful to consider a wider range of parameters than adopted by Kepner et al. (1997). We find below that the magnitude of $b$ is a crucial factor in regulating the star formation efficiency in dwarf galaxies. 
In the analysis of Kepner et al. (1997), gas is assumed to be in quasi-hydrostatic equilibrium at all times. Yet, both pre-star formation evolution and post-star formation feedback processes lead to flow and evolution of the residual gas mass that should be treated with a time-dependent dynamical calculation. In addition, external perturbations, such as tidal disturbances by nearby large galaxies or collisions with other gas-rich dwarf galaxies are also important in modifying the density and velocity of the gas. For some models, we approximate the possibility of a strong tidal compression (or a mild collision) with an initial velocity perturbation in the form of a Hubble inflow

$$
v_{i}=-H_{p} r
$$

When scaled with the halo radius and the characteristic velocity dispersion, the above expression is equivalent to $v_{i} / v_{0}=-h_{p}\left(r / r_{\text {halo }}\right)$.

The main purpose of our calculation is to study the conditions under which old stars formed within dwarf galaxies. The age of these stars, 10-12 Gyr, corresponds to high-red-shift epochs, when the external UV flux is intense. We approximate the frequency dependence of the external UV energy flux by

$$
J_{\nu}(z)=J_{0}(z)\left(\nu / \nu_{0}\right)^{-\alpha} .
$$

For the isotropic external flux, the rate at which photons enter the dwarf galaxy (photons/s), whose outer surface area is $S$, is

$$
Q_{e x t}=S \times \int_{\nu_{0}}^{\infty} \frac{2 \pi J_{\nu}}{h \nu} d \nu=\frac{2 \pi J_{0} S}{h \alpha} .
$$

The flux of photons across the surface would involve integrating over $\mu \mathrm{d} \mu$ where $\mu=\cos \theta$, and $\theta$ is the angle from the normal. The resulting value of $Q_{e x t}$ would be smaller by a factor of two. This introduces some uncertainty into our results, but is smaller than the uncertainty in the magnitude of the UV background, and much smaller than the range in $Q_{\text {ext }}$ experienced by dwarf galaxies over their lifetimes. We adopt $\alpha=1.5$ in all our calculations.

Ionizing photons are also generated by the massive stars formed within these galaxies. The UV production rate by massive stars in each zone (photons/s) is

$$
Q_{\star j}=N_{\star j} \bar{Q}_{\star},
$$

where $N_{\star j}$ is the number of massive stars of zone, and $\bar{Q}_{\star}$ is the value of $Q_{\star}$ averaged over massive stars according to the Miller-Scalo IMF $\left(\bar{Q}_{\star}=10^{48.5} \mathrm{~s}^{-1}\right)$.

\subsection{The Basic Models: Effect of Baryonic Fraction}

\subsubsection{Dwarf Galaxies with Modest Velocity Dispersion}

The parameters of the models that we have examined are shown in Table 1. In the table are listed, for each model, the scale radius $\left(r_{0}\right)$, the initial baryonic-to-dark matter ratio $(b)$, the ratio of the background flux to its value at the current epoch, the upper limit upon the star formation efficiency $\left(\beta_{\max }\right)$, the falue of $f_{I M F}$, the value of $\tau_{\text {onset }}$, and the fraction of gas converted into stars, $f_{\star}$.

In the first series of three models, A1, A2, and A3, we simulate the evolution of an unperturbed dwarf galaxy $\left(H_{p}=0\right)$ at the present epoch $\left(J_{0}=4 . \times 10^{-23} \mathrm{erg} \mathrm{s}^{-1} \mathrm{~cm}^{-2} \mathrm{ster}^{-1} \mathrm{~Hz}^{-1}\right)$. The scale length of 
the potential $r_{0}=0.6 \mathrm{kpc}$, such that $M_{0}=2.2 \times 10^{7} M_{\odot}, v_{0}=12.6 \mathrm{~km} \mathrm{~s}^{-1}$, and $M_{D M}=1.3 \times 10^{8} M_{\odot}$. These model parameters for the halo strcuture are comparable to those of Leo I and other dSph (Mateo et al. 1998). In order to demonstrate the dependence of the star formation efficiency on the ordinary matter content, we consider $b=0.001,0.1$, and 0.2 . In these and all other models, the ordinary matter has the same initial spatial density distribution as the dark matter $\left(\rho_{g, i}=b \rho_{D M}\right)$, and an initial temperature of $10 \mathrm{~K}$ everywhere.

In Figure 3, we plot the fraction, $f_{\star}$, of ordinary mass that is converted into stars as a function of time. For the tenuous ordinary matter distribution in model A1, the residual $\mathrm{H}$ is fully ionized, and no stars are formed. Despite the low initial temperature, the residual gas is ionized and heated to $T=1.5 \times 10^{4} \mathrm{~K}$ almost instantly. As the gas evolves toward a new equilibrium, most of it leaves the galaxy. For the residual gas, the recombination process is inefficient compared with the photoionization even for the present low flux of background ionizing photons.

For models A2 and A3, the outer regions of the galaxy (beyond 0.6 and $1 \mathrm{kpc}$ respectively), quickly become photoionized while the gas that is initially located near the central regions of the galaxy collapses to form stars with $f_{\star}=3.6 \times 10^{-2}$ and $5.2 \times 10^{-2}$. The total stellar content is

$$
M_{\star}=f_{\star} b M_{D M},
$$

so that the total mass of the stars formed in these two models are $4.6 \times 10^{5} M_{\odot}$ and $1.3 \times 10^{6} M_{\odot}$, comparable to stellar content of the Ursa Minor and Leo I dSph respectively. The results of these models indicate that the hydrogen gas has sufficiently large column density to become self-shielded against the external UV flux if the ionization fraction is low. But, in both models, the onset of star formation quickly leads to photoionization from stellar UV emission, and an outward expansion of the gas. In the shallow potential of low-velocity dwarf galaxies, a large fraction of the ionized gas escapes from the system. Eventually, the residual gas evolves toward new equilibria, with a density that is too low for effective recombination. Consequently, an inflated, hot, and highly ionized state is attained, in which star formation is quenched (Figure 4).

The comparison between models A1, A2, and A3 indicates that unperturbed dwarf galaxies with very low ordinary matter contents are likely to remain fully ionized. Nevertheless, a small population of stars may be formed within these galaxies if a critical amount of gas can become neutral. The conversion efficiency is a function of the baryonic mass. Hydrogen may become neutral either due to initial conditions, by accretion of additional gas from the intergalactic medium, or it may be induced by cloud-cloud collisions (see below). Although mass loss can reduce $M_{\star} / M_{D M}$ to well below both its initial value and the cosmological $\Omega_{b} / \Omega_{d}$, the amount of stars formed in these systems provide a lower limit upon its initial value, $b$.

\subsubsection{Very Low Mass Dwarf Galaxies}

Although the potential in models A1, A2, and A3 is relatively small, most dwarf spheroidal galaxies have even shallower potentials and smaller velocity dispersions. In order to assess the efficiency of initial star formation in such systems, we consider models B1, B2, and B3, in which $r_{0}$ is set to be 0.3 , such that $v_{0}=8.5 \mathrm{~km} \mathrm{~s}^{-1}, M_{0}=4.3 \times 10^{6} M_{\odot}$, and $M_{D M}=2.5 \times 10^{7} M_{\odot}$. The value of $b$ for these three models are $0.1,0.2$, and 0.5 , so that the total baryonic mass in model B3 equals that in model A2. The external UV flux all three models is $J_{0}=4 . \times 10^{-23} \mathrm{erg} \mathrm{s}^{-1} \mathrm{~cm}^{-2} \mathrm{ster}^{-1} \mathrm{~Hz}^{-1}$.

In comparison with model A2, model B3 is more compact with a higher $\rho_{0}$ and more rapid decline in the density distribution. Although the actual baryonic mass contained in the initial Strömgren radius in 
model B3 is smaller than that in model A2, the gravitational potential for $r_{0}=0.3$ is shallower than that for $r_{0}=0.6$. In both models, the gas was not in a hydrostatic equilibrium initially. Because the gas collapses faster in the more massive model A2 than in model B3, a slightly larger fraction of the gas was turned into stars during the initial formation episode.

The subsequent evolution of the gas and stars in models B1, B2, and B3 are similar to those in models A2 and A3. After an initial episode of star formation, the residual gas becomes fully ionized, a substantial amount of mass is lost, and star formation is quenched. The asymptotic values of $f_{\star}$ for these models are plotted in Figure 5a. The trend of $f_{\star}$ increasing with $b$ generally holds. The magnitude of $M_{\star}$ is $8 \times 10^{3} M_{\odot}$ and $2 \times 10^{4} M_{\odot}$ for models B2 and B3 respectively. Thus, even with $b$ substantially larger than the cosmological $\Omega_{b} / \Omega_{d}$, the mass of stars formed is substantially below the stellar contents in dSphs.

\subsubsection{Star Formation at Early Epochs}

In the above models, we adopt the background UV flux for low redshift. The old stellar population in dSphs was, however, formed at high redshifts, when the background UV flux was considerably more intense. In models C1, C2, and C3 (Figure 5b), we adopt $J_{0}=4 \times 10^{-21} \mathrm{ergs} \mathrm{s}^{-1} \mathrm{~cm}^{-2} \mathrm{ster}^{-1} \mathrm{~Hz}^{-1}$, with $r_{0}=0.3$, and, respectively, $b=0.1,0.2$, and 0.5. All other model parameters are similar to those in the B series of models. Although $J_{0}$ in models $\mathrm{C} 1, \mathrm{C} 2$ and $\mathrm{C} 3$ is $10^{2}$ larger than that in the previous models, the resulting $f_{\star}$ and hence $M_{\star}$ for models B3 and C3 are comparable to each other. The density at the Strömgren surface is proportional to $J_{0}^{1 / 2}$, and so the C-series of models are photoionized closer to the galaxy center. But the initial episode of star formation in both model B3 and C3 is quenched primarily by the feedback, rather than by photoionization from external UV radiation. The difference in $f_{\star}$ is substantial, however, between the most tenuous models, B1 and $\mathrm{C} 1$. The value of $J_{0}$ is sufficient to completely ionize the gas in model C1 prior to the onset of collapse. Consequently, the star formation efficiency is essentially zero for model C1.

\subsection{Effect of Gravitational Potential}

\subsubsection{Star Formation at Recent Epochs}

The models considered above have relatively shallow gravitational potentials, such that the residual gas can escape after it is ionized. We now consider a series of models with deeper gravitational potentials. In models D1, D2, D3, D4, and D5, we adopt, respectively, $r_{0}=0.3,0.6,2,4$, and 6 . We set $b=0.1$ for all five models. In each, we also use $J_{0}=4 \times 10^{-23} \mathrm{ergs} \mathrm{s}^{-1} \mathrm{~cm}^{-2} \mathrm{ster}^{-1} \mathrm{~Hz}^{-1}$, such that models D1 and D2 are identical, respectively, to models B1, and A2. For models D3, D4, and D5, respectively, $M_{0}=3.6 \times 10^{8} M_{\odot}$, $1.8 \times 10^{9} M_{\odot}$, and $4.7 \times 10^{9} M_{\odot} ; M_{D M}=2.1 \times 10^{9} M_{\odot}, 10^{10} M_{\odot}$, and $2.7 \times 10^{10} M_{\odot} ;$ and $v_{0}=28,45$, and $58 \mathrm{~km} \mathrm{~s}^{-1}$. The potentials of these models are deeper than those of the dSphs but comparable to those of typical dwarf elliptical and dwarf irregular galaxies.

In Figure $6 \mathrm{a}$, we plot the evolution of $f_{\star}$ for various models. These results clearly indicate that the residual gas is retained by the gravitational potential of the dwarf galaxies in models D4 and D5. Star formation in each of these cases is self-regulated. In model D4, the mass of the stellar component reaches $M_{\star} \sim 6.4 \times 10^{8} M_{\odot}$ with a comparable amount of residual gas bound to the galaxy after 1 Gy. Thereafter,

star formation continues at a self-regulated rate of $\sim 0.2 \mathrm{M}_{\odot} \mathrm{yr}^{-1}$. For the more massive model D5, more than half of its original gas is converted into stars relatively early. After $300 \mathrm{Myr}$, the mass of the stars 
becomes $2 \times 10^{9} \mathrm{M}_{\odot}$ with $\sim 5 \times 10^{8} \mathrm{M}_{\odot}$ of residual gas. The residual gas is much more extended than originally, which slows down the star formation rate.

\subsubsection{Star Formation at Early Epochs}

The external UV flux in models D1-5 corresponds to the present-day value. In order to consider the conditions under which stars as old as 10-12 Gyr formed, we consider models E1-5 (Figure 6b). These models are identical to models D1-5, except that $J_{0}$ is increased by two orders of magnitude (i.e. $J_{0}=$ $\left.4 \times 10^{-21} \mathrm{erg} \mathrm{s}^{-1} \mathrm{~cm}^{-2} \mathrm{ster}^{-1} \mathrm{~Hz}^{-1}\right)$. With this enhanced UV flux, the gas in model E2 is ionized immediately, and no star formation occurs. By contrast, the lower UV flux present in model D1 allows for the initial formation of a few stars.

The intense UV radiation increases the density needed for ionization equilibrium, and so the radius of the Strömgren sphere is reduced. This effect is most evident in the marginal models D3 and E3. Photoionization due to the background is weak in the low $J_{0}$ model D3. In that model, the residual gas undergoes rapid collapse, leading to high early levels of star formation and feedback, resulting in two early, strong 'bursts' of star formation. The momentum injected to the gas by the star formation is sufficient to drive the gas outwards, such that it becomes too tenuous to recombine, and subsequent star formation is quenched. In contrast, the outer regions of model E3 are ionized. But the gravitational potential is sufficiently deep to retain the ionized gas. Star formation is therefore able to proceed, and does so at a nearly constant rate, regulated by the supernova injection of momentum, and reduced relative the initial rate of model D3 due to the higher level of ionization. Somewhat counterintuitively, therefore, model E3 by late times is able to convert a higher fraction of its gas into stars than is model D3. In Figure 7, we show the density profile snapshots of model D3 (solid lines) and E3 (dotted lines) at $t=30,100,150$ and 300 Myr.

Evolution of the star formation rate for the more massive models E4 and E5 are nearly indistinguishable from those of D4 and D5. This implies that the rich supply of gas in both cases limits the effects of the external UV radiation to the gas in the tenuous outer halo, well outside of the central, star-forming regions, which have much shorter recombination and dynamical timescales.

In order to verify this tendency, we examine models F1-F4, in all four of which $b=0.01$. In models F1 and $\mathrm{F} 2, r_{0}=4 \mathrm{kpc}$, whereas it is $6 \mathrm{kpc}$ for models F3 and F4. The external $J_{0}=4 \times 10^{-23} \mathrm{erg} \mathrm{s}^{-1} \mathrm{~cm}^{-2} \mathrm{ster}^{-1} \mathrm{~Hz}^{-1}$ for models F1 and F3, whereas $J_{0}=0$ for models F2 and F4. The evolution of $f_{\star}$ for all four models is shown in Figure 8. The comparison between models F1 and F2 (as well as that between F3 and D4) indicates that the external flux does not significantly modify the star formation rate. The nearly steady star formation rate is self-regulated.

\subsection{Star Formation Efficiency}

The above results indicate that, in systems with $r_{0}<2 \mathrm{kpc}$, the residual gas expands towards a new equilibrium after an initial epoch of star formation. Consequently, mass is lost from the galaxy and the residual gas density becomes sufficiently depleted that the external background UV flux can severely suppress the star formation rate. The magnitude of $f_{\star}$ may be enhanced if the feedback effect is weaker than in the standard models. Such might be the case if $\beta_{\max }$ is relatively small (corresponding to an inefficient conversion of gas into stars), or if $f_{I M F}$ is relatively large (corresponding to a suppressed production rate of 
the massive supernovae progenitor stars). In models G1-G4, we consider some variations of model A3 (which is also referred to as G0 for comparison purposes). In each, $J_{0}=4 \times 10^{-23} \mathrm{erg} \mathrm{s}^{-1} \mathrm{~cm}^{-2} \mathrm{ster}^{-1} \mathrm{~Hz}^{-1}, b=0.2$, and $r_{0}=0.6 \mathrm{kpc}$. In model G1, we set $\beta_{\max }=0.1$ and $f_{I M F}=1$. In models G2 and G3, we set $\beta_{\max }=1$ and $f_{I M F}=10$, while in model G4, $\beta_{\max }=0.1$ and $f_{I M F}=10$. In models G0-2, $\tau_{\text {onset }}=0.5 \mathrm{Myr}$, but in models G3 and G4, $\tau_{\text {onset }}=5$ Myr (see Fig. 9). The magnitude of $f_{\star}$ for model G3 is almost an order of magnitude larger than that of model G0 (A3). In comparing between models G1 and G2, we see that $f_{\star}$ depends more sensitively on the value of $\beta_{\max }$ than upon $f_{I M F}$ and $\tau_{\text {onset }}$.

\subsection{Self-regulated, Episodic Star Formation}

Star formation in the more massive galaxies, with larger $r_{0}$, discussed above is self-regulated by the feedback process. In the models presented so far, self-regulated star formation leads to quasi-equilibria, such that the star formation rate is essentially constant. For models D4, D5, E4, and E5 there are small variations in the star formation rate during the early evolution of the galaxy. In these models, the star formation rate is relatively steady, because the local values of $\tau_{f f}+\tau_{\text {onset }}$ are much shorter than the average dynamical timescale of the star forming region, which itself is regulated by the feedback process. In contrast, for model D3, with a more compact structure, the time delay can lead to episodic variations in the star formation rate. However, the supply of baryonic mass is limited, and after one or two episodes of star formation the density is reduced sufficiently that the residual gas remains ionized and star formation is quenched.

Some dwarf galaxies show evidence for episodic star formation histories. In order for episodic star formation to take place, three conditions must be satisfied: 1) the galaxy must have sufficient baryonic mass to become self-shielded against the background UV radiation, 2) the collapse and feedback switch-on delay times must be longer than the global dynamical timescale, in order that a substantial amount of baryonic matter may accumulate prior to the onset of each episode of star formation, and 3) the star formation efficiency must be modest, so that an adequate amount of residual gas may be preserved for the subsequent episodes of star formation. Models H1-H5, have the same values of $r_{0}\left(=4 \mathrm{kpc}\right.$ ), and $J_{0}$ as model D4 (which we aso refer to as the fiducial model $\mathrm{H} 0$ ). In models $\mathrm{H} 1$ and $\mathrm{H} 2$, we set $\tau_{\text {onset }}=5 \mathrm{My}$ and $50 \mathrm{My}$ respectively. In both cases, the delay in the switch on of the feedback effects allows more material to accumulate into the central regions of the galaxy. The delay enhances the eventual rate of star formation leading to ferocious feedback, driving all the residual gas out of the galaxies.

In models $\mathrm{H} 3$ and $\mathrm{H} 4$, we set, respectively, $\beta_{\max }=0.1$ and $10^{-2}$, motivated to satisfy requirement 3 ) above for episodic star formation. In Figure 10, we see that these models indeed lead to episodic variations in the star formation rate. Finally, in model H5, we also include the effect of a bottom-heavy initial mass function by setting $f_{I M F}=10$. In this case, a large fraction of the ordinary matter is retained by the galaxies, and converted into stars.

\subsection{Effect of External Perturbations}

One difficulty associated with the results presented above is the low gas-to-star conversion efficiency for the lowest mass dwarf galaxies. In addition to reducing the severity of the feedback effect, we now explore the possibility of compressions, such as would result from either tidal perturbations or collisions with other galaxies. The models which we consider are shown in Table 2. In that table are listed, for each model, the values of $r_{0}, b, J_{0}(z) / J_{0}(0), H_{p} / H_{0}, f_{I M F}, \tau_{\text {onset }}$, and $f_{\star}$. The value of $H_{p}$ indicates the strength of a 
linear compression imposed upon the models, as described in Equation 3-3, and is normalized in the table to $H_{0}=1 \mathrm{~km} \mathrm{~s}^{-1} \mathrm{kpc}^{-1}$.

Models I1-I4 have values of $J_{0}, r_{0}$, and $b$ identical to those of model A3. In models I1-I4, respectively, $H_{p}=3,15,30$, and $150 \mathrm{~km} \mathrm{~s}^{-1} \mathrm{kpc}^{-1}\left(h_{p}=0.49,2.4,4.9\right.$, and 24). The compressional timescale across the galaxies, $r_{\text {halo }} / H_{p}=1,0.2,0.1$, and $0.02 \mathrm{Gyr}$, respectively, for models I1-4. For the region which contains most of the gas in models I3 and I4, the compressional speed is supersonic and the density jump at the shock fronts induces large increases in the local star formation rate and efficiency. Consequently, $f_{\star}$ is substantially enhanced.

The results are shown in Figure 11, where it can clearly be seen that $f_{\star}$ is an increasing function of $H_{p}$. The enhancement is associated with the increased densities, and resulting enhanced recombination rates, which provide a better shield for the residual gas in the galaxy. We note that the compression is excessive for model I4. Such a large impact is more appropriate for cloud-cloud collisions, rather than tidal compression. These results indicate a significant fraction of the baryons in dwarf galaxies can be converted into stars if the galaxies are subjected to supersonic compressions.

Finally, we consider the effect of impact on relatively massive dwarf galaxies similar to models D3 and $\mathrm{E} 3$ (which is refereed to as models $\mathrm{K} 0$ and $\mathrm{K} 2$ here). We compare the results of these models without external perturbation to those of models $\mathrm{K} 1$ and $\mathrm{K} 3$ with a modest external perturbation $\left(H_{p}=3 \mathrm{~km} \mathrm{~s}^{-1} \mathrm{kpc}^{-1}\right)$. Both K0 and K1 have an external UV flux of $J_{0}=4 \times 10^{-23} \mathrm{erg} \mathrm{s}^{-1} \mathrm{~cm}^{-2} \mathrm{ster}^{-1} \mathrm{~Hz}^{-1}$; while both $\mathrm{K} 2$ and $\mathrm{K} 3 J_{0}=4 \times 10^{-21} \mathrm{erg} \mathrm{s}^{-1} \mathrm{~cm}^{-2} \mathrm{ster}^{-1} \mathrm{~Hz}^{-1}$. The results are shown in Figure 12. For models $\mathrm{K} 1$ and K3, the compression speed is slightly slower than the sound speed of the ionized gas in the outer regions of the galaxies. Nevertheless, it can confine the thermally expanding ionized shell. Thus, the compressional speed not only leads to a density enhancement, but also retention of the gas, enabling the gas in model K1 to undergo protracted and self-regulated star formation. We also note that even with such a modest compressional speed, gas in these dwarf galaxies can recombine efficiently even in early cosmological epoches when the intensity of the UV flux is relatively high. The comparison between models K0 and K1 indicates that this compression can double the star formation efficiency.

\section{Summary and Discussion}

In this paper, we consider a series of one-dimensional numerical models to examine the efficiency of star formation in dwarf galaxies under the influence of local cooling, heating by UV photons from both the background radiation and hot stars, and the winds and supernovae associated with massive stars. Radiative transfer is handled using a simple "Strömgren shell" approximation. Completely ionized gas is assumed to have temperature of $15,000 \mathrm{~K}$, while heating and cooling are followed dynamically in gas that is not completely ionized. The effects of supernovae and winds from massive stars are included in the momentum equation of the gas. Stars can form in gas that has cooled sufficiently for the Jeans mass to be within the range of stellar masses, which is not tidally limited, and for which the free-fall timescale is short compared to the pre-main sequence evolution time of massive stars, $\tau_{\text {onset }}$. The rate of conversion of gas into stars is a fraction of the gas free-fall timescale. Feedback from the massive stars which form is delayed by the free-fall time, plus $\tau_{\text {onset }}$.

The most important parameters explored in the models are: 1) the depth of the galactic potential, 2) the baryonic fraction, 3) the background UV flux, 4) $\left.\tau_{\text {onset }}, 5\right)$ the IMF, and 6) compressional perturbation (see Tables 1 and 2). 


\subsection{The Initial Baryonic-to-Dark Matter Ratio}

We initially confirm the results of Kepner et al (1997), that for a low mass system $\left(M_{D M} \lesssim 10^{8} \mathrm{M}_{\odot}\right)$ with baryonic fraction $b=0.001$, even today's background UV flux is adequate to totally ionize the gas such that no stars can form. With increasing baryonic fraction, however, these systems eventually become self-shielded from the background UV, and the gas is able to cool and collapse, leading to star formation. For $b=0.2$ (comparable to the ratio of $\Omega_{b} / \Omega_{d}$ today), a low mass system with $r_{0}=0.3 \mathrm{kpc}$ is able to convert $\sim 2 \%$ gas $\left(\sim 10^{5} M_{\odot}\right)$ into stars in about 2 billion years. Feedback from star formation leads to rapid expansion of the gas within the shallow galactic potential. The lower recombination efficiency of the expanded gas allows it to be completely ionized by the external UV radiation, and star formation is terminated after the initial, weak burst. Modest retention of gas is possible, if the IMF of the stars formed in these system is biased towards the low-mass stars.

In the presence of much stronger background UV radiation, appropriate to early epochs, star formation may still occur in small galaxies having $b \gtrsim 0.2$. In such systems, self-shielding is sufficient to prevent ionization initially within the cores, even with the higher UV background. Star formation then proceeds, governed solely by internal time scales and feedback, while the external radiation acts only at late times, to prevent further star formation. The requirement for a relatively large concentration of ordinary matter in these shallow potential is in contrast to the low- $b$ estimate made by Kepner et al (1997) based on the assumption of Bondi accretion into dark matter potentials. Although the accretion process may be more effective when the pristine gas is cold before the epoch of reionization, all present-day dwarf galaxies must have acquired their ordinary matter content prior to the formation of massive stars in other nearby dwarf galaxies. The relatively short dynamical time scales within the dwarf galaxies suggests that such a precise timing may be difficult to achieve. Alternatively, prior to the epoch of dwarf galaxy formation, density fluctuation of the baryonic matter may be well correlated and coupled to that of the dark matter.

\subsection{Mass-to-Light Ratio}

Star formation is much more efficient in higher mass systems, due both to increased self-shielding and to the enhanced ability of the galactic potential to retain ionized gas. A ten-fold increase in the dark matter mass, from $10^{7-8}$ typical of dSphs, to $10^{8-9}$ typical of dwarf Ellipticals (dEs) or dwarf Irregulars (dIrrs), may lead to an order of magnitude increase in the gas-to-star conversion efficiency. In the most massive systems, the star formation rate is self-regulated by internal feedback, independent of the external UV flux. For marginal systems, with low external flux, star formation occurs in a rapid initial burst, followed by expansion and ionization by the external flux, similar to the low-mass systems. The presence of a higher external UV flux, however, lessens the initial star formation rate, reducing the subsequent feedback, and allowing star formation to proceed at a slow pace for an extended period.

Observational data suggest that in the satellite dSphs around the Galaxy, the baryonic matter content varies widely, while the dark matter not only dominates but also has widely varying masses. But, in dwarf galaxies with mass more than $\sim 10^{7-8} M_{\odot}$, the total mass-to-light ratio has a nearly constant value $\sim 2.0$ (see Mateo et al. 1998). The results of our calculation are consistent with this apparent dichotomy. In order to make a direct comparison with Fig. 7 in Mateo et al. (1998), we plot the total mass to light ratio $M / L$ within $r_{0}$ as a function of the absolute visual magnitude $M_{v}$ of the different dwarf galaxies in models D1-5, A1-3, and G0-5. For very low-mass $\left(M_{0}<5 \times 10^{6} M_{\odot}\right)$ dark matter halo, star formation may be completely

suppressed even if $b$ is 0.5 . In these low-mass systems, the pressure scale height of the photoionized gas is 
comparable to the galaxy's radius and in the vicinity of the Galactic disks, and the tidal perturbation of nearby host galaxy would induce efficient mass loss (Murray et al. 2003). It is entirely possible that that the halo of the Milky Way may be populated by many dark matter halos of the 'missing dwarf galaxies'. For the intermediate-mass $\left(M_{0} \sim 10^{7-8}\right)$ dwarf galaxies, the gas retention efficiency depends on many factors such as the background UV flux, the IMF, the baryonic to dark matter ratio etc. In Figure 13, the $(M / L)-M_{v}$ correlation for this mass range merely indicate that a transition mass for the gas retention efficiency. For the high-mass dwarf galaxies, the baryonic matter represents a significant fraction of the total mass and the constant $M / L$ is a reflection of the self-regulated star formation process.

\subsection{Multiple Bursts of Star Formation}

In higher mass systems, feedback occurs before the gas can evolve significantly. Star formation is therefore able to occur at an almost equilibrium, self-regulated rate.

By contrast, in low mass systems, $\tau_{f f}+\tau_{\text {onset }}$ exceeds the dynamical time on which the gas evolves within the galactic potential. If sufficient gas is present to shield the core from external UV, then gas in the core may accumulate significantly before the onset of feedback, leading to strong bursts of star formation. The same is observed in larger systems, for which we have considered larger values of $\tau_{\text {onset }}$.

If the initial burst of star formation causes the gas to expand, and its density to decrease to the point at which the external UV is able to ionize the galaxy completely, then star formation is terminated after a single burst. In somewhat more massive systems, gas in the core is still self-shielded after the massive stars formed in the initial burst fade away. The gas can then cool, contract, and trigger additional bursts of star formation, as is observed in some of our models. In all systems which undergo either one or multiple episodes of star formation, the feedback is eventually sufficiently strong as to drive the gas out of the system.

Episodic star formation may also result from reducing the efficiency of feedback, either by reducing the conversion efficiency of gas into stars, or the fraction of massive stars which form. In high mass models with such variations, we observe episodic star formation.

\subsection{Induced Star Formation}

Within the environment of a galaxy cluster, or near to a massive galaxy, a dwarf system shall experience tidal forces, that shall vary over time, resulting in occasional, or even periodic compressions. If dwarf systems form in large numbers around more massive overdense regions, they shall also experience collisions. The merger process which builds larger galactic entities can also lead to collisions and disturbances.

Both mechanisms lead to enhancements in the density, and therefore the recombination efficiency of the gas within dwarf galaxies. In small systems at early epochs, this may allow the galaxy to form some stars, when it might otherwise be completely ionized by the external UV radiation. In more massive systems, the star formation rates are enhanced by as much as an order of magnitude relative to unperturbed systems for realistic compression speeds.

Perturbations such as considered here would be expected to be stronger in dense cluster environments, or near to massive galaxies. In the former, collisions would be more frequent, while in the latter, tidal variations due to elliptical orbits around the parent system are strongest. These are also environments 
where tidal stripping, ram pressure, and external UV radiation are all enhanced, which would be expected to act to reduce star formation efficiencies, and so it is unclear whether the effect of perturbations alone would lead to environmental dependences for stellar populations within dwarf galaxies.

\subsection{Consequences for Dwarf Galaxy Evolution}

As indicated in earlier work, star formation in the lowest mass dwarf galaxies is difficult at early epochs, and the observed population of dSphs appear to be at the very edge of the ability of small galaxies to form stars. The difficulty is due to the strong UV background, which easily ionizes the galaxies throughout, preventing the gas from cooling and contracting within the weak gravitational potential of the dwarf system.

Star formation may still occur, even in the smallest systems, however, if the gas within them can become self-shielded from the external UV. Such might be the case if they form with a relatively large baryonic fraction, if they accrete additional gas, or if they undergo compressions due to either tidal forces or collisions. Such processes may help to explain the existence of old stellar populations in even the smallest dSphs. Feedback from star formation also forms a natural means for avoiding the "over-cooling" problem among the dwarf progenitors of massive galaxies.

In small systems, star formation tends to occur in bursts, the feedback from which either expels the gas completely from the galaxy, or drives it to large radii, making it vulnerable to either tidal or ram-pressure stripping. Subsequent generations of star formation would, therefore, appear to be unlikely. The gas lost to a dwarf system, however, leaves at small speeds $\left(\sim 10 \mathrm{~km} \mathrm{~s}^{-1}\right)$. It shall not, therefore, move to large distances from the dwarf as it orbits within the potential of either a massive galaxy or within a cluster, and may be re-accreted by the dwarf at apogalacticon, possibly leading to subsequent bursts of star formation. Some small dwarf systems, therefore, might be expected to show periodic bursts of star formation, with a burst period determined by the orbital period of the dwarf.

By contrast, in larger systems, where the feedback time is short compared to the dynamical time of the system, and where the potential is capable of retaining ionized gas, feedback tends to lead to steady, self-regulated star formation. The gas within the system does, however, expand under the influence of stellar feedback, and so may also be subject to stripping within galactic cluster environments (Murray, Dong, \& Lin 2003).

The possibility of severe mass loss from dwarf galaxies following star formation indicates that the currently observed baryonic-to-dark matter ratios can provide only lower limits, possibly very weak limits, to the values present when the galaxies formed. Our models indicate that the star formation efficiencies, and the ability of galaxies to retain gas following star formation depend very sensitively dependences upon the evolutionary histories of the galaxies, and upon the galactic potentials, which may account for the large dispersion in the stellar to dark-matter fraction observed today among the dwarf galaxies.

We thank Drs. A. Burkert, and K-S. Oh for useful conversations. We also thank the anonymous referee for valuable comments. This work was performed under the auspices of the U.S. Department of Energy by University of California, Lawrence Livermore National Laboratory under Contract W-7405-Eng-48. This work is partially supported by NASA through an astrophysical theory grant NAG5-12151. 


\section{REFERENCES}

Anninos, P., Norman, M. L., \& Clarke, D. A. 1994, ApJ, 436, 11

Bechtold, J., Weymann, R. J., Lin, Z., \& Malkan, M. A. 1987, ApJ, 315, 180

Blumenthal, G. R., Faber, S. M., Primack, J. R., \& Rees, M. J. 1984, Nature, 311, 517

Bonnell, I. A., Bate, M. R., \& Zinnecker, H. 1998, MNRAS, 298, 93

Bowen, D. V., Tolstoy, E., Ferrara, A., Blades, J. C., \& Brinks, E. 1997, ApJ, 478, 530

Burkert, A. 1995, ApJ, 447, L25

Carignan, C., Beaulieu, S., Côte, S., Demers, S., \& Mateo, M. 1998, AJ, 116, 1690

Dalgarno, A., \& McCray, R. A. 1972, ARAA, 10, 375

de Bernardis, P., et al 2002, ApJ, 564, 559

Dekel, A. \& Silk, J. 1986, ApJ, 303, 39

Elmegreen, B.G. \& Lada, C. 1977, ApJ, 214, 725

Fall, S. M. \& Rees, M. J. 1985, ApJ, 298, 18

Grebel, E. K. 1997, Rev. Mod. Astronomy, 10, 29

Grebel, E. K., \& Stetson, P.B. 1998, in IAU Symposium 192-The Stellar Content of Local Group Galaxies, ed. P. Whitelock \& R. Cannon, (San Francisco: ASP), 11

Haardt, F., \& Madau, P. 1996, ApJ, 461, 20

Haiman, Z., Rees, M. J., \& Loeb, A. 1996, ApJ, 467, 522

Haiman, Z., Thoul, A. A., \& Loeb, A. 1996, ApJ, 464, 523

Kang, H., Shapiro, P. R., Fall, S. M., \& Rees, M. J. 1990, ApJ, 363, 488

Katz, N., Weinberg, D. H., \& Hernquist, L. 1996, ApJS, 105, 19

Kepner, J. V., Babul, A., \& Spergel, D. N. 1997, ApJ, 487, 61

Klypin, A. A., Kravtsov, A. V., Valenzuela, O. \& Prada, F. 1999, ApJ, 522, 82

Klypin, A. A., Nolthenius, R., \& Primack 1997, ApJ, 516, 530

Knapp, G. R., Kerr, F. J. \& Bowers, P. F. 1978, AJ, 83, 360

Lin, D. N. C., \& Murray, S. D. 1992, ApJ, 394, 523

Mateo, M. 1998, ARA\&A, 36, 435

Mateo, M., Olszewsky, E.W., Vogt, S.S., \& Keane, M.J. 1998, AJ, 116, 2315

Miller, G. E. \& Scalo, J. M. 1979, ApJS, 41, 513 
Moore, B., Ghigna, F., Governato, F., Lake, G., Stadel, J. \& Tozzi, P. 1999, ApJ, 524, L19

Mori, M. \& Burkert, A. 2000, ApJ, 538, 559

Mould, J. R., Bothun, G. D., Hall, P. J., Staveley-Smith, L., \& Wright, A. E. 1990, ApJL, 362 , L55

Murray, S. D., Dong, S., \& Lin, D. N. C. 2003, ApJ, in press

Murray, S. D. \& Lin, D. N. C. 1996, ApJ, 467, 728

Navarro, J. F. \& Benz, W. 1991, ApJ, 380, 320

Navarro, J. F., Frenk, C. S., \& White, S. D. M. 1995, MNRAS, 275, 56

Navarro, J. F. \& Steinmetz, M. 1997, ApJ, 478, 13

Navarro, J. F. \& White, S. D. M. 1993, MNRAS, 265, 271

Noriega-Crespo, A., Bodenheimer, P., Lin, D. N. C., \& Tenorio-Tagle, G. 1989, MNRAS, 237,46

Osterbrock, D. E. 1989, Astrophysics of Gaseous Nebulae and Active Galactic Nuclei (Mill Valley: University Science Books)

Richtmyer, R. D., \& Morton, K. W. 1967, Difference Methods for Initial-Value Problems (Interscience Publishers)

Scalo, J. M. 1986, Fund. Cosmic Phys., 11, 1

Sommer-Larsen, J., Gelato, S. \& Vedel, H. 1999, ApJ 519, 501

Weil, M. L., Eke, V. R. \& Efstathiou, G. 1998, MNRAS 300, 773

Weinberg, D. H., Hernquist, L., \& Katz, N. 1997, ApJ, 477, 8

White, S. D. M. \& Rees, M. J. 1978, MNRAS, 183, 341

Yorke, H. W. \& Sonnhalter, C. 2002, ApJ, 569, 846

This preprint was prepared with the AAS $\mathrm{LAT}_{\mathrm{E}} \mathrm{X}$ macros v5.0. 
Table 1. Static Galaxy Models

\begin{tabular}{|c|c|c|c|c|c|c|c|}
\hline Model & $\begin{array}{c}\mathrm{r}_{0} \\
(\mathrm{kpc})\end{array}$ & $b$ & $\frac{J_{\nu}(z)}{J_{\nu}(0)}$ & $\beta_{\max }$ & $f_{I M F}$ & $\begin{array}{l}\tau_{\text {onset }} \\
(\mathrm{Myr})\end{array}$ & $\begin{array}{c}f_{*} \\
(\%)\end{array}$ \\
\hline $\mathrm{A} 1$ & 0.6 & 0.001 & 1 & 1.0 & 1 & 0.5 & 0.0 \\
\hline A2 & 0.6 & 0.1 & 1 & 1.0 & 1 & 0.5 & 2.6 \\
\hline A3 & 0.6 & 0.2 & 1 & 1.0 & 1 & 0.5 & 3.7 \\
\hline B1 & 0.3 & 0.1 & 1 & 1.0 & 1 & 0.5 & 1.3 \\
\hline B2 & 0.3 & 0.2 & 1 & 1.0 & 1 & 0.5 & 1.9 \\
\hline B3 & 0.3 & 0.5 & 1 & 1.0 & 1 & 0.5 & 2.5 \\
\hline $\mathrm{C} 1$ & 0.3 & 0.1 & 100 & 1.0 & 1 & 0.5 & 0.0 \\
\hline $\mathrm{C} 2$ & 0.3 & 0.2 & 100 & 1.0 & 1 & 0.5 & 0.7 \\
\hline C3 & 0.3 & 0.5 & 100 & 1.0 & 1 & 0.5 & 1.5 \\
\hline D1 & 0.3 & 0.1 & 1 & 1.0 & 1 & 0.5 & 1.3 \\
\hline D2 & 0.6 & 0.1 & 1 & 1.0 & 1 & 0.5 & 2.6 \\
\hline D3 & 2.0 & 0.1 & 1 & 1.0 & 1 & 0.5 & 14.2 \\
\hline D4 & 4.0 & 0.1 & 1 & 1.0 & 1 & 0.5 & 68.4 \\
\hline D5 & 6.0 & 0.1 & 1 & 1.0 & 1 & 0.5 & 83.2 \\
\hline E1 & 0.3 & 0.1 & 100 & 1.0 & 1 & 0.5 & 0.0 \\
\hline $\mathrm{E} 2$ & 0.6 & 0.1 & 100 & 1.0 & 1 & 0.5 & 0.0 \\
\hline E3 & 2.0 & 0.1 & 100 & 1.0 & 1 & 0.5 & 19.7 \\
\hline $\mathrm{E} 4$ & 4.0 & 0.1 & 100 & 1.0 & 1 & 0.5 & 66.3 \\
\hline E5 & 6.0 & 0.1 & 100 & 1.0 & 1 & 0.5 & 67.6 \\
\hline F1 & 4.0 & 0.01 & 0 & 1.0 & 1 & 0.5 & 24.5 \\
\hline $\mathrm{F} 2$ & 4.0 & 0.01 & 1 & 1.0 & 1 & 0.5 & 24.1 \\
\hline F3 & 6.0 & 0.01 & 0 & 1.0 & 1 & 0.5 & 47.7 \\
\hline F4 & 6.0 & 0.01 & 1 & 1.0 & 1 & 0.5 & 45.4 \\
\hline G0 & 0.6 & 0.2 & 1 & 1.0 & 1 & 0.5 & 3.7 \\
\hline G1 & 0.6 & 0.2 & 1 & 0.1 & 1 & 0.5 & 3.5 \\
\hline G2 & 0.6 & 0.2 & 1 & 1.0 & 10 & 0.5 & 11.7 \\
\hline G3 & 0.6 & 0.2 & 1 & 1.0 & 1 & 5.0 & 6.3 \\
\hline G4 & 0.6 & 0.2 & 1 & 0.1 & 10 & 5.0 & 22.4 \\
\hline $\mathrm{H} 0$ & 4.0 & 0.1 & 1 & 1.0 & 1 & 0.5 & 68.4 \\
\hline H1 & 4.0 & 0.1 & 1 & 1.0 & 1 & 5.0 & 71.7 \\
\hline $\mathrm{H} 2$ & 4.0 & 0.1 & 1 & 1.0 & 1 & 50. & 35.0 \\
\hline H3 & 4.0 & 0.1 & 1 & 0.1 & 1 & 5.0 & 69.7 \\
\hline $\mathrm{H} 4$ & 4.0 & 0.1 & 1 & 0.01 & 1 & 5.0 & 49.4 \\
\hline H5 & 4.0 & 0.1 & 1 & 0.1 & 10 & 5.0 & 85.7 \\
\hline
\end{tabular}


Table 2. Externally Perturbed Galaxy Models

\begin{tabular}{cccccccc}
\hline \hline Model & $\begin{array}{c}\mathrm{r}_{0} \\
(\mathrm{kpc})\end{array}$ & $b$ & $\frac{J_{\nu}(z)}{J_{\nu}(0)}$ & $H_{p} / H_{0}$ & $f_{I M F}$ & $\begin{array}{c}\tau_{\text {onset }} \\
(\mathrm{Myr})\end{array}$ & $\begin{array}{c}f_{*} \\
(\%)\end{array}$ \\
\hline I0 & 0.6 & 0.1 & 1 & 0 & 1 & 0.5 & 3.7 \\
I1 & 0.6 & 0.1 & 1 & 3 & 1 & 0.5 & 4.2 \\
I2 & 0.6 & 0.1 & 1 & 15 & 1 & 0.5 & 6.3 \\
I3 & 0.6 & 0.1 & 1 & 30 & 1 & 0.5 & 27.3 \\
I4 & 0.6 & 0.1 & 1 & 150 & 1 & 0.5 & 89.3 \\
K0 & 2.0 & 0.1 & 1 & 0 & 1 & 0.5 & 14.2 \\
K1 & 2.0 & 0.1 & 1 & 3 & 1 & 0.5 & 32.1 \\
K2 & 2.0 & 0.1 & 100 & 0 & 1 & 0.5 & 19.7 \\
K3 & 2.0 & 0.1 & 100 & 3 & 1 & 0.5 & 23.7 \\
\hline
\end{tabular}




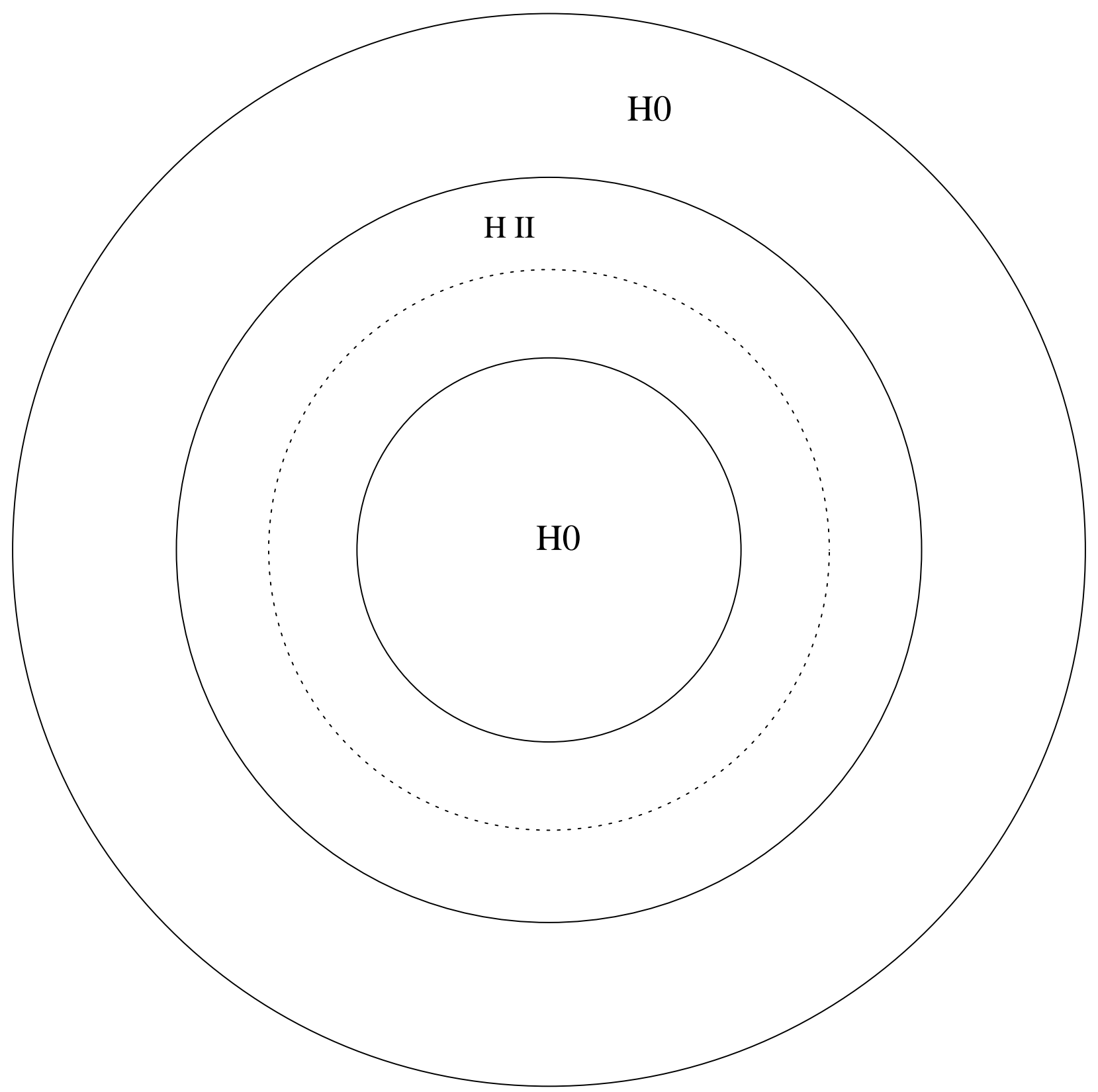

Fig. 1.- Strömgren shell model. 


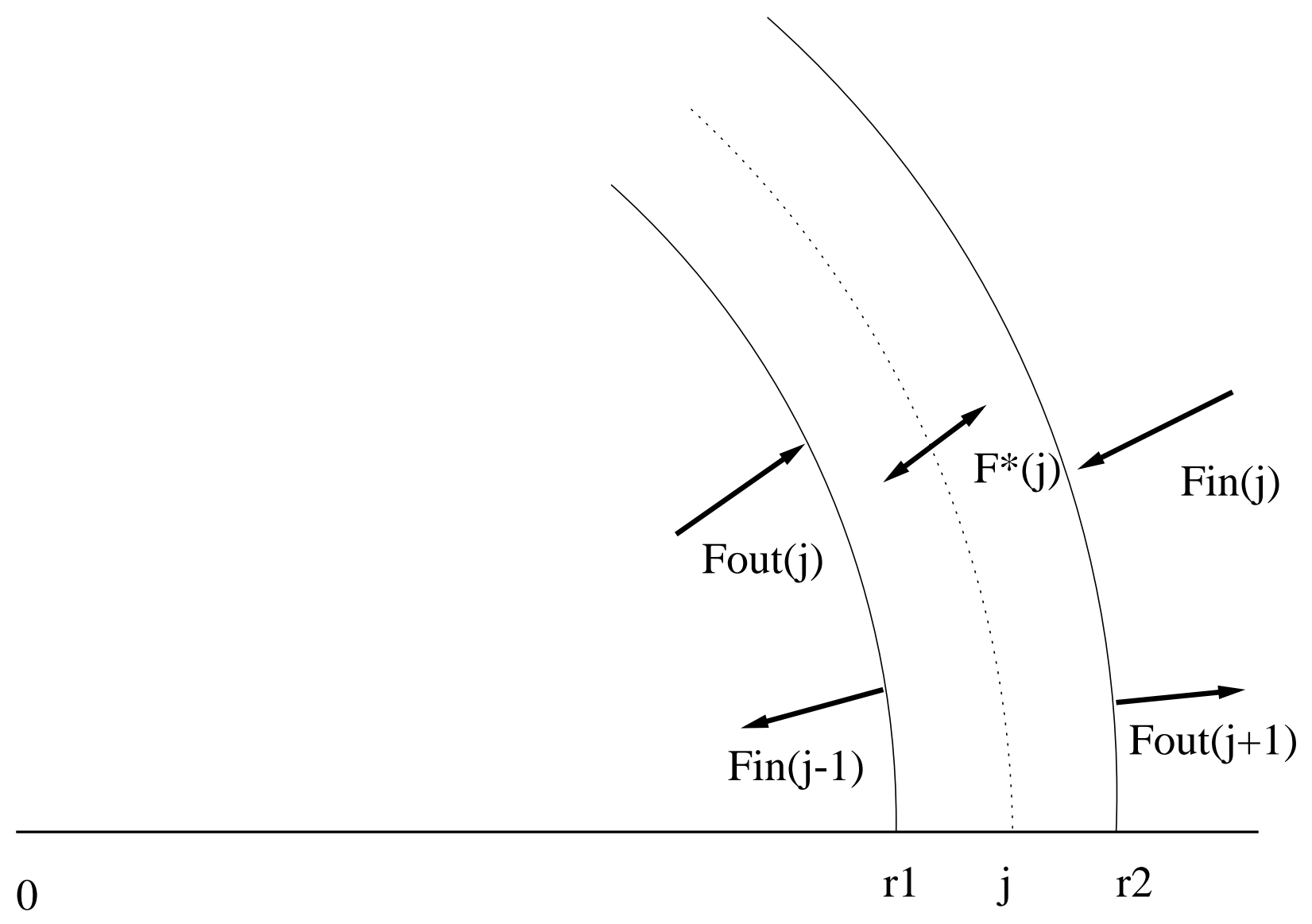

Fig. 2.- Radiative transfer at zone j. 


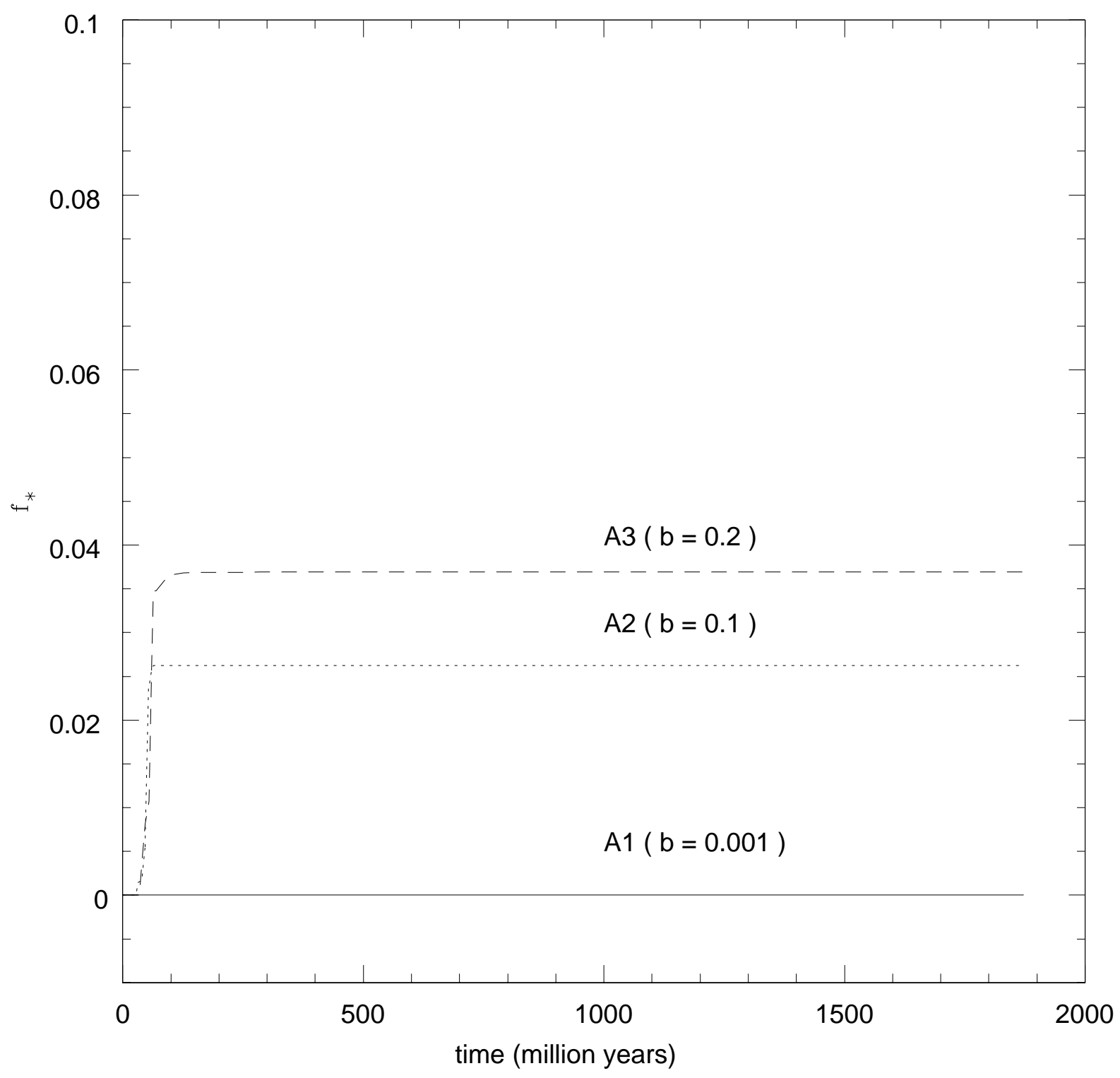

Fig. 3. - Star formation history for models with $r_{0}=0.6 \mathrm{kpc}, J_{0}=4 \times 10^{-23}$, and $b=0.001,0.1$ and 0.2 respectively for model A1, A2 and A3. 


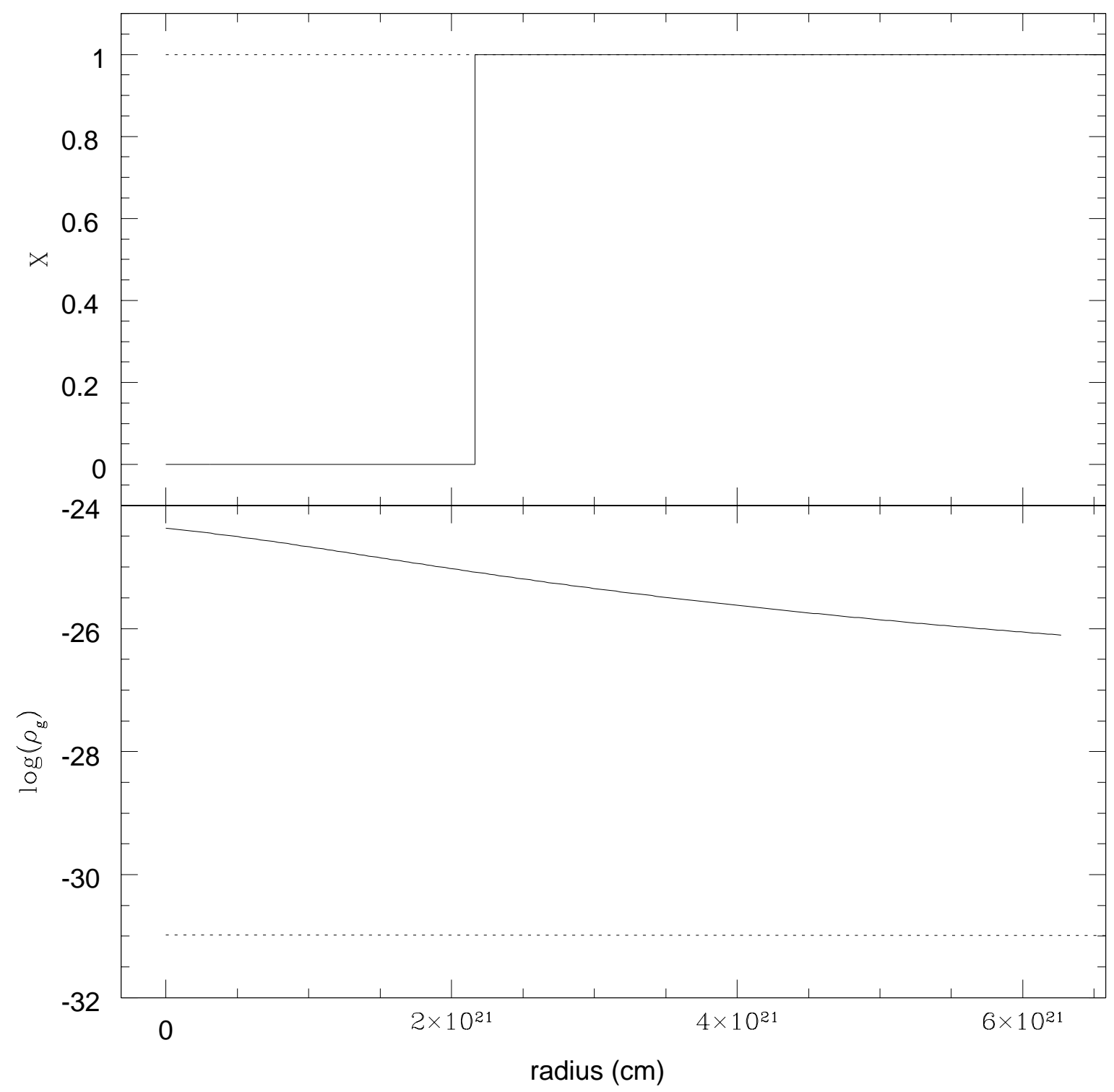

Fig. 4.- Ionization fraction and density profile for the initial (solid lines) and final (dotted lines) states of model A2. 


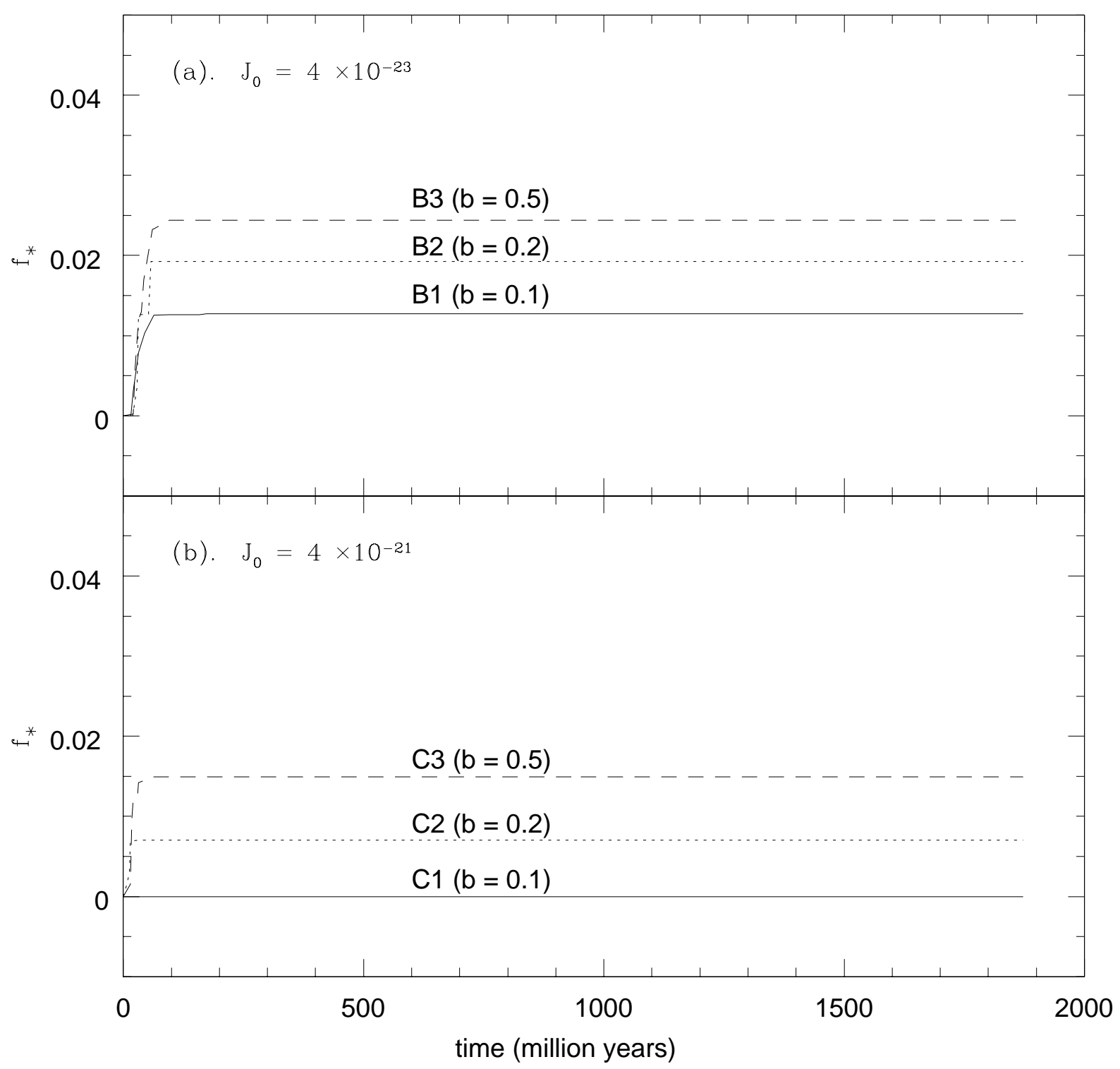

Fig. 5. - Star formation history for models with $r_{0}=0.3 \mathrm{kpc}$ : (a). $J_{0}=4 \times 10^{-23}$, and $b=0.001,0.1$ and 0.2 for model B1, B2 and B3 respectively; (b). $J_{0}=4 \times 10^{-21}$, and $b=0.001,0.1$ and 0.2 for model C1, C2 and C3 respectively. 


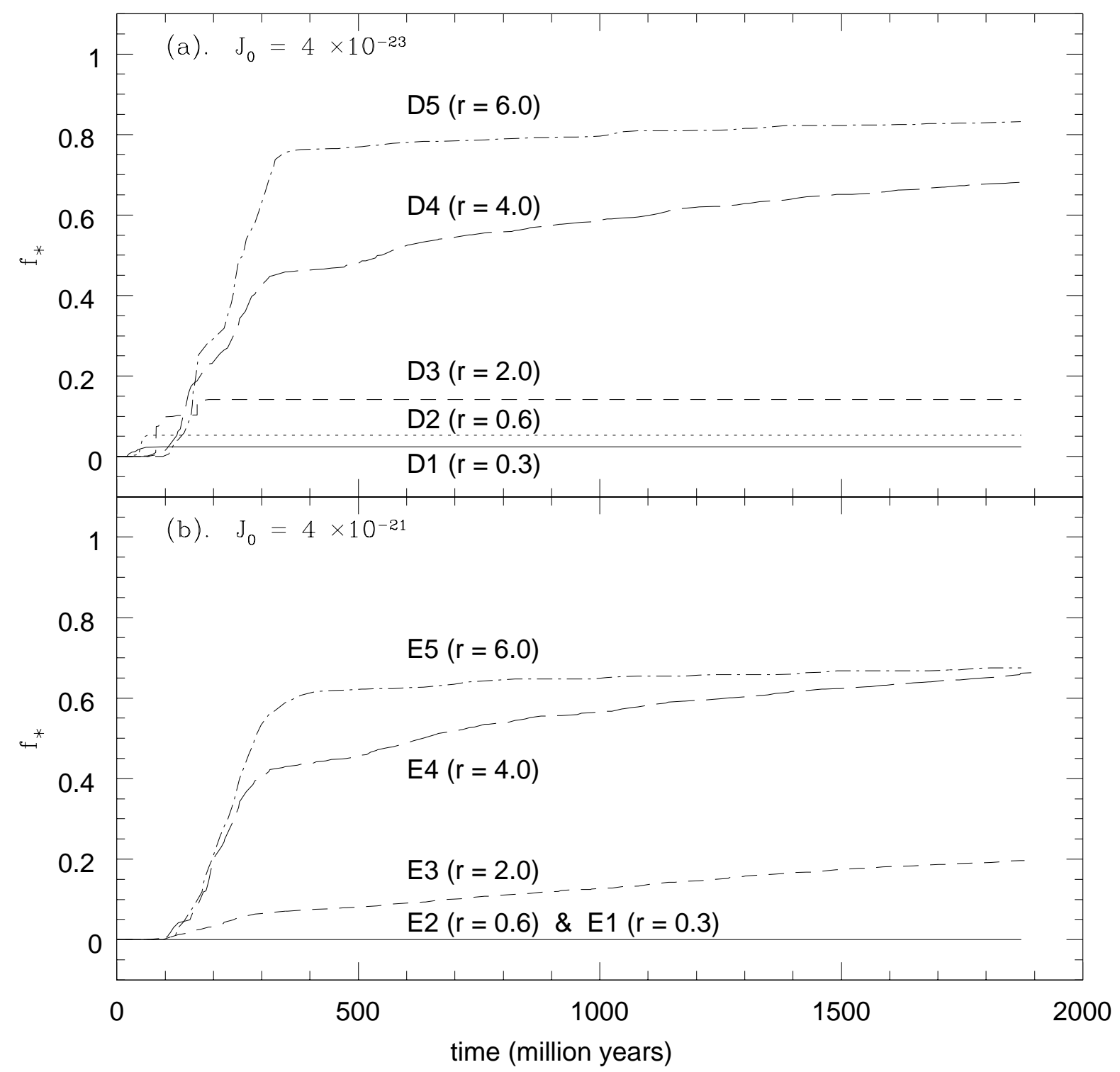

Fig. 6. - Star formation history for models with $b=0.1$ : (a). $J_{0}=4 \times 10^{-23}$, and $r_{0}=0.3,0.6,2.0,4.0$ and 6.0 for model D1, D2, D3, D4 and D5 respectively; (b). $J_{0}=4 \times 10^{-21}$, and $r_{0}=0.3,0.6,2.0,4.0$ and 6.0 for model E1, E2, E3, E4 and E5 respectively. 


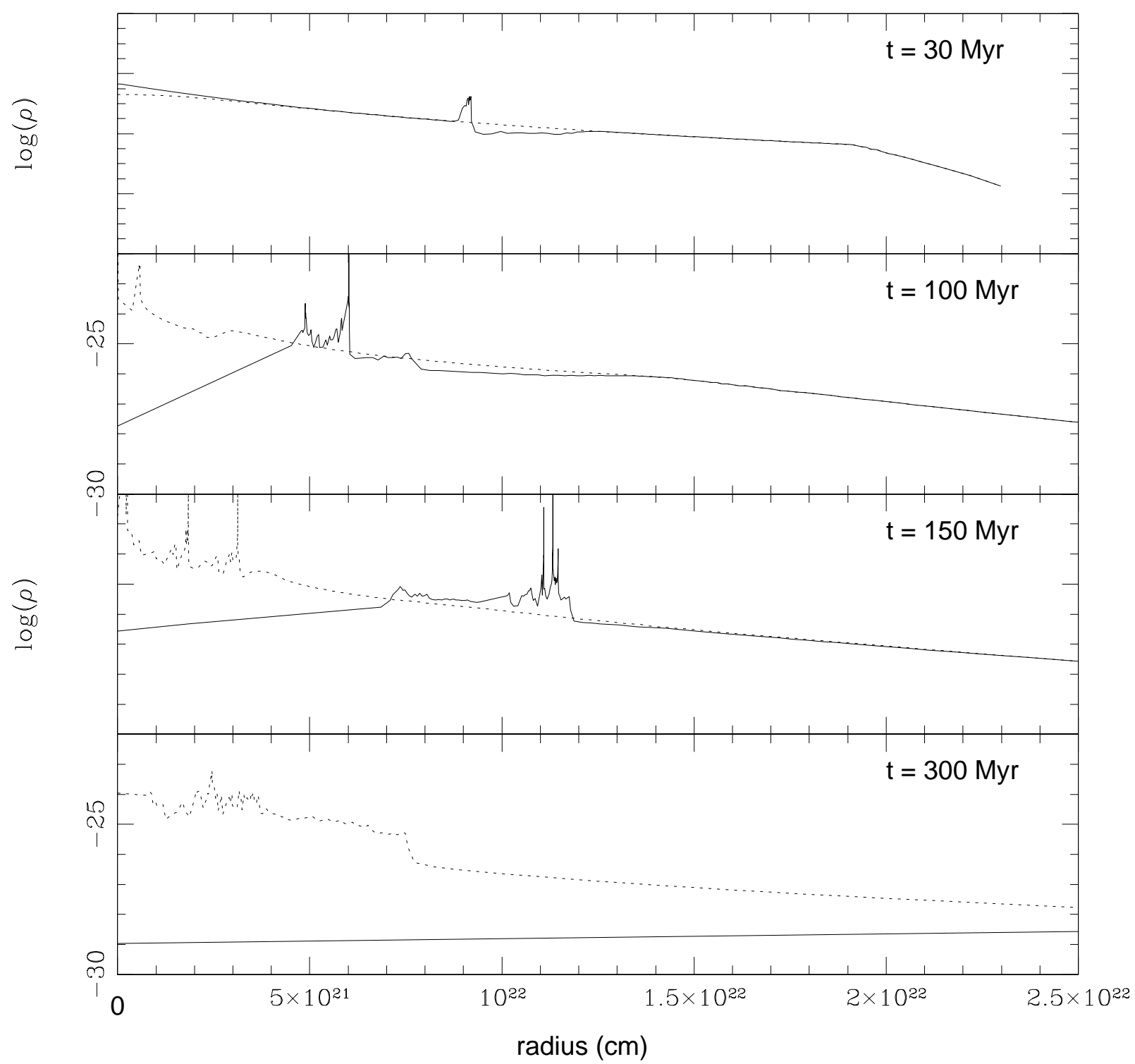

Fig. 7.- Gas density profile snapshots for model D3 (solid lines) and E3 (dotted lines) at $t=30,100,150$ and $300 \mathrm{Myr}$. 


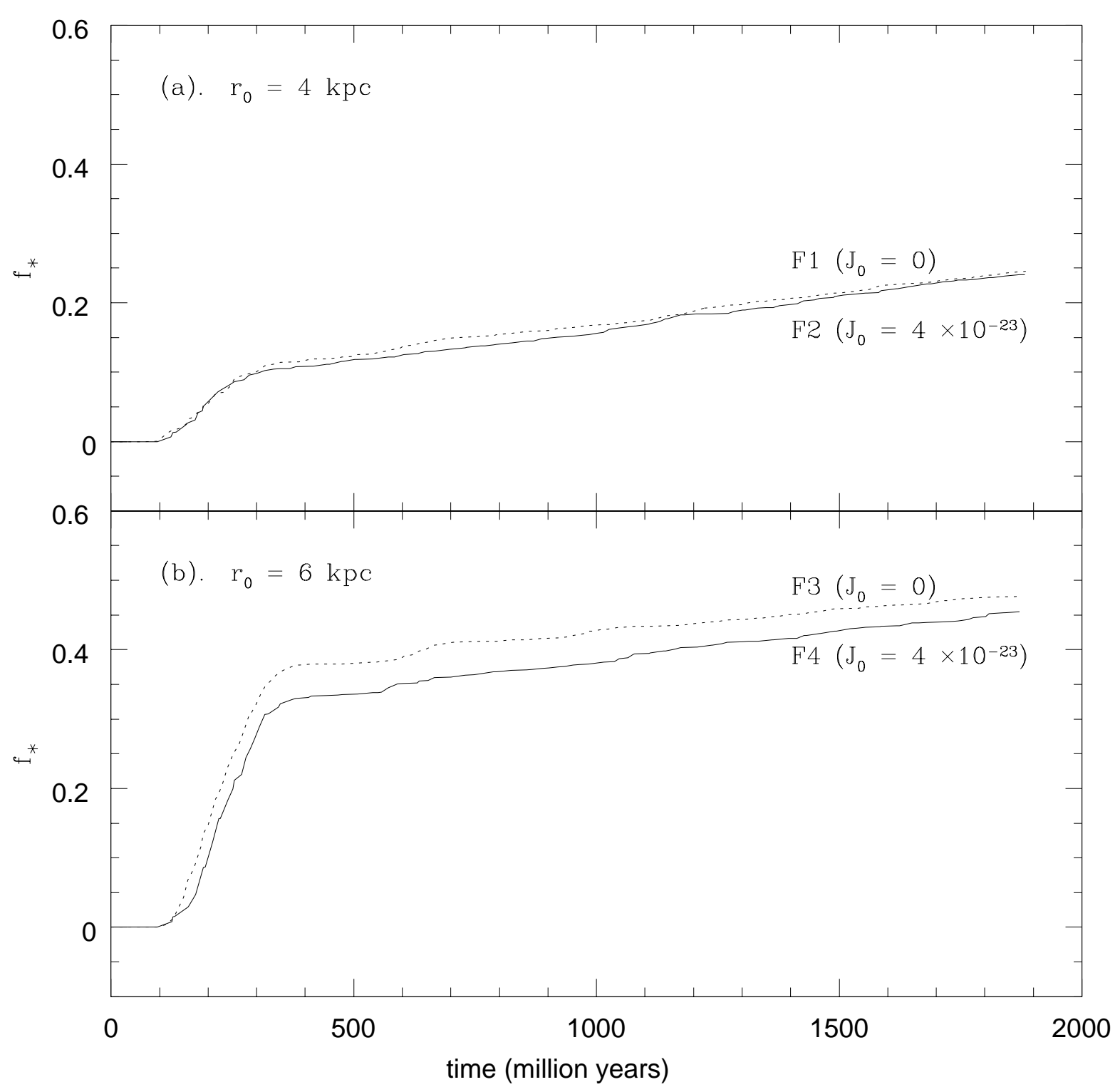

Fig. 8. - Star formation history for models with $b=0.01$. (a). $r_{0}=4.0$, and $J_{0}=0,4 \times 10^{-23}$ for model $\mathrm{F} 1, \mathrm{~F} 2$ respectively; (b). $r_{0}=6.0$, and $J_{0}=0,4 \times 10^{-23}$ for model F3, F4 respectively. 


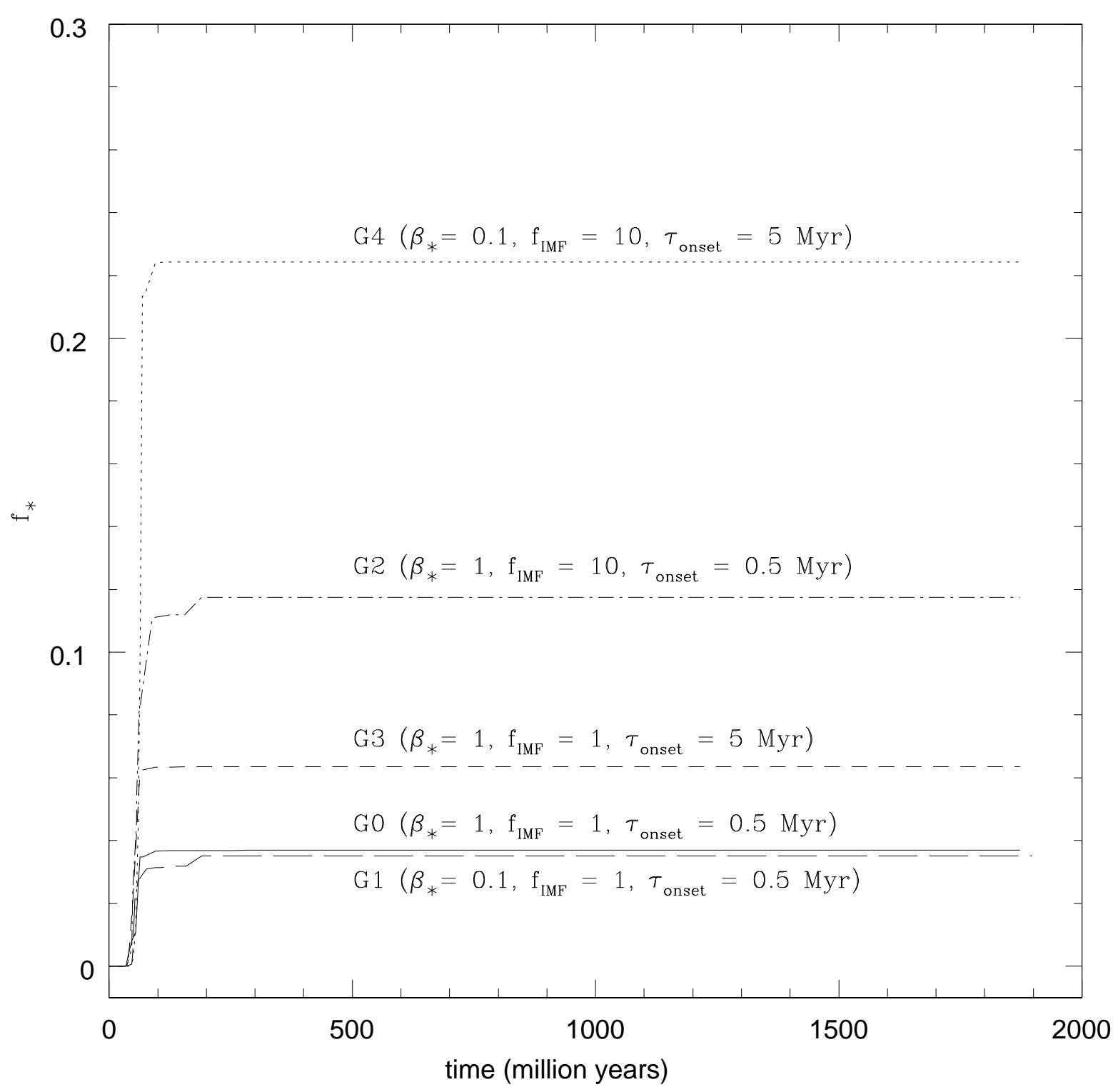

Fig. 9.- Star formation efficiency. All models with $r_{0}=0.6 \mathrm{kpc}, J_{0}=4 \times 10^{-23}$, and $b=0.2$, but with different parameters for star formation efficiency. Model G0 (base model): $\beta_{\max }=1, f_{I M F}=1$, $\tau_{\text {onset }}=0.5 \mathrm{Myr} ;$ Model G1: $\beta_{\max }=0.1, f_{I M F}=1, \tau_{\text {onset }}=0.5 \mathrm{Myr} ;$ Model G2: $\beta_{\max }=1, f_{I M F}=10$, $\tau_{\text {onset }}=0.5 \mathrm{Myr}$; Model G3: $\beta_{\max }=1, f_{I M F}=1, \tau_{\text {onset }}=5 \mathrm{Myr} ;$ Model G4: $\beta_{\max }=0.1, f_{I M F}=10$, $\tau_{\text {onset }}=5 \mathrm{Myr}$ 


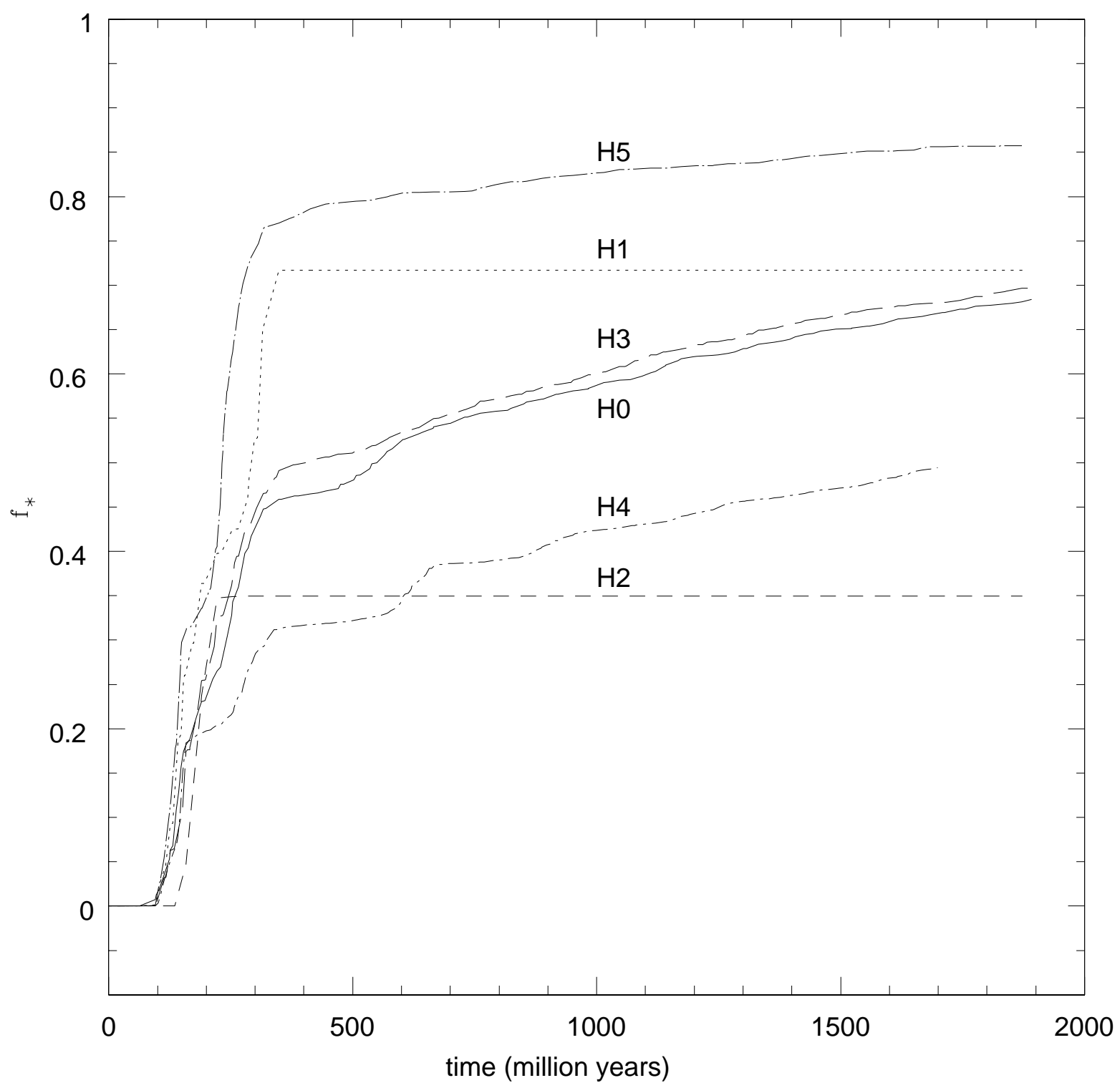

Fig. 10. - Star formation efficiency. All models with $r_{0}=4.0 \mathrm{kpc}, J_{0}=4 \times 10^{-23}$, and $b=0.1$, but with different parameters for star formation efficiency. Model H0 (base model): $\beta_{\max }=1, f_{I M F}=1$, $\tau_{\text {onset }}=0.5 \mathrm{Myr} ;$ Model H1: $\beta_{\max }=1, f_{I M F}=1, \tau_{\text {onset }}=5 \mathrm{Myr} ;$ Model H2: $\beta_{\max }=1, f_{I M F}=1$, $\tau_{\text {onset }}=50$ Myr; Model H3: $\beta_{\max }=0.1, f_{I M F}=1, \tau_{\text {onset }}=5$ Myr; Model H4: $\beta_{\max }=0.01, f_{I M F}=1$, $\tau_{\text {onset }}=5 \mathrm{Myr} ;$ Model H5: $\beta_{\max }=0.1, f_{I M F}=10, \tau_{\text {onset }}=5 \mathrm{Myr}$ 


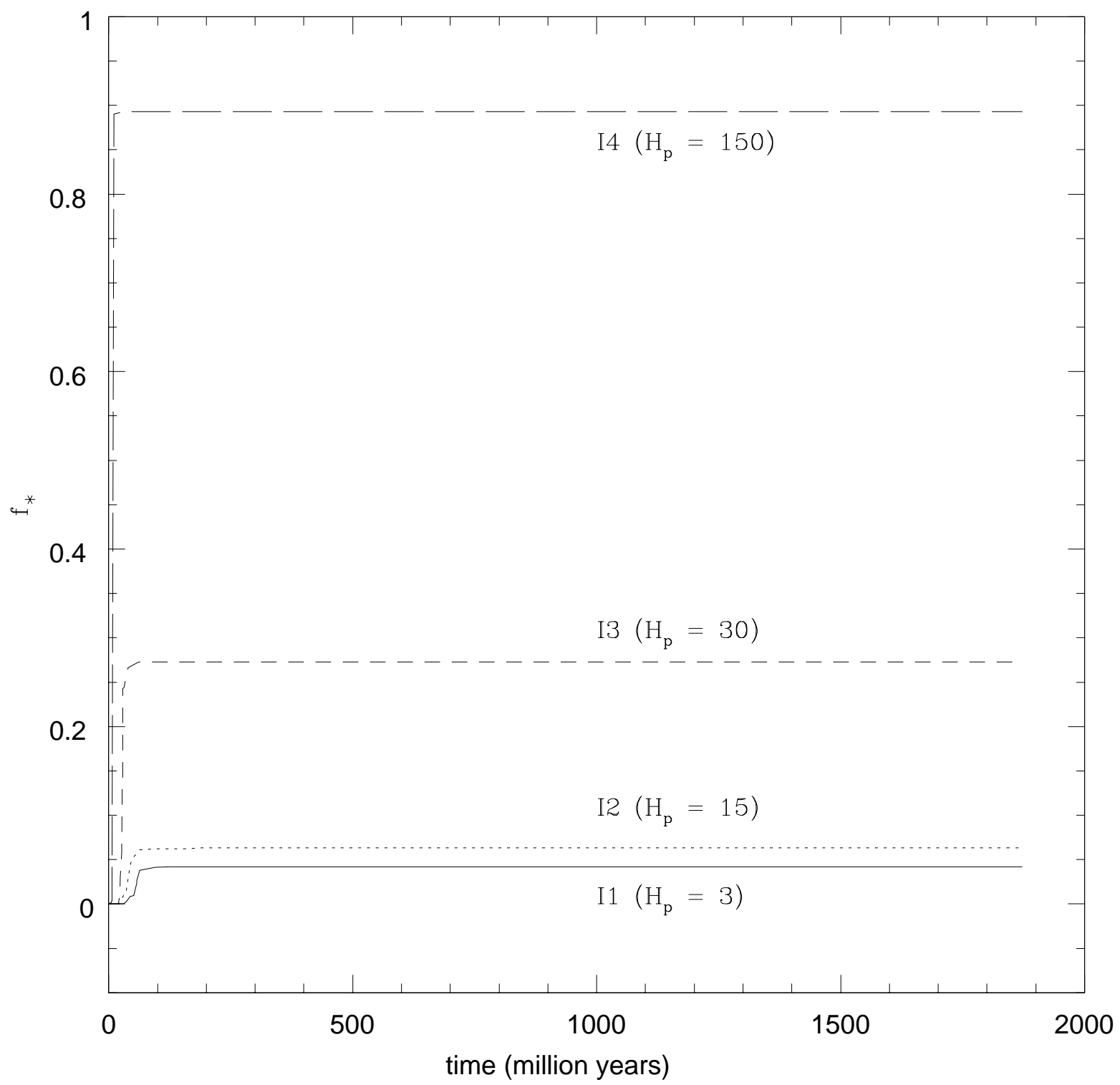

Fig. 11. - Star formation history for models with external perturbation. All models with $r_{0}=0.6 \mathrm{kpc}$, $J_{0}=4 \times 10^{-23}$, and $b=0.2$, but with different external perturbation strength: $H_{p}=3,15,30$, and $150 \mathrm{~km} \mathrm{~s}^{-1} \mathrm{kpc}^{-1}$ for models I1-I4 respectively. 


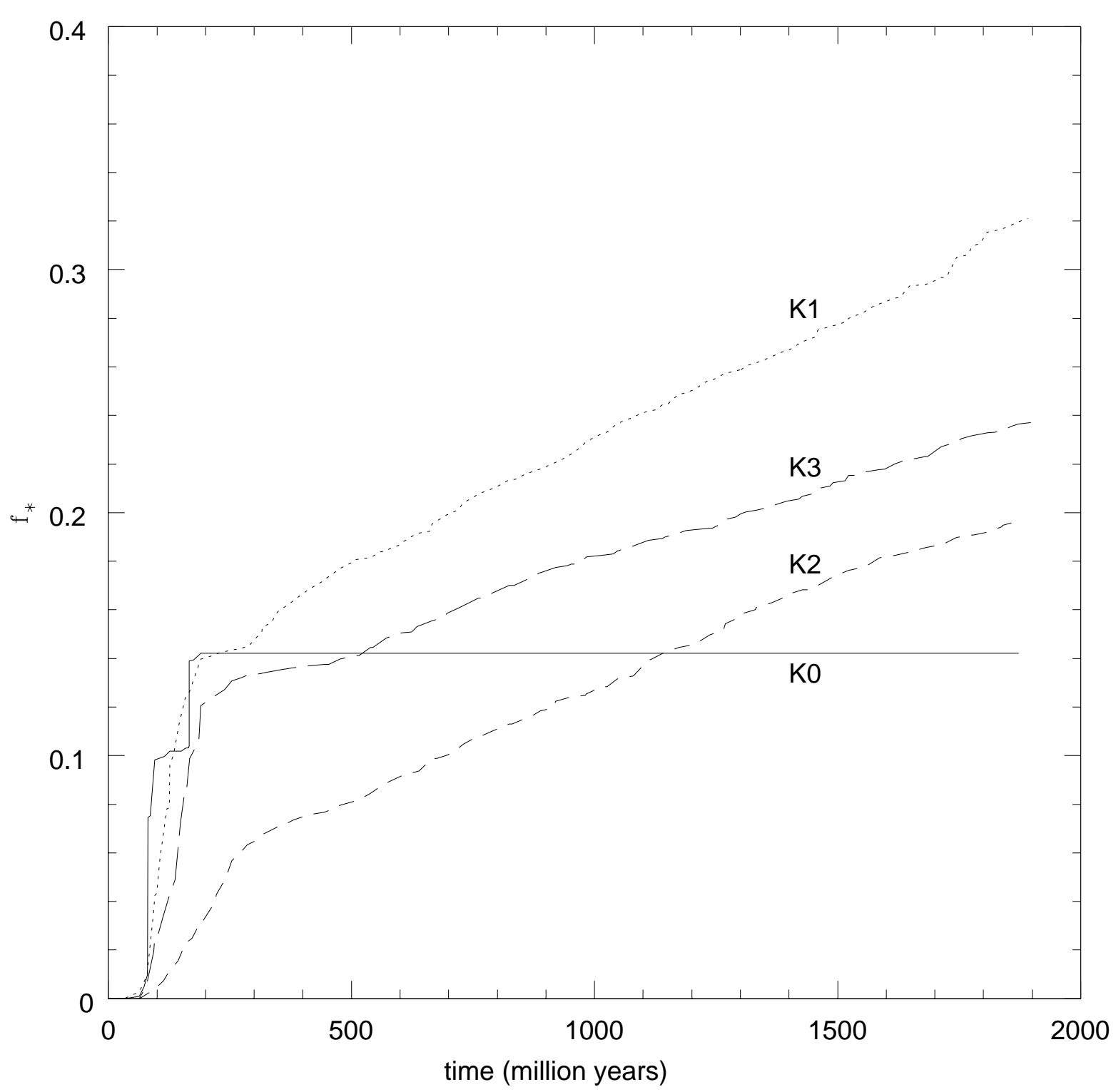

Fig. 12. - Effect of external perturbation. All models with $r_{0}=2 \mathrm{kpc}$, and $b=0.1$. Same $J_{0}=4 \times 10^{-23}$, but different $H_{p}=0$ and 3 for models K0 and K1 respectively; Same $J_{0}=4 \times 10^{-21}$, but different $H_{p}=0$ and 3 for models $\mathrm{K} 2$ and K3 respectively. 


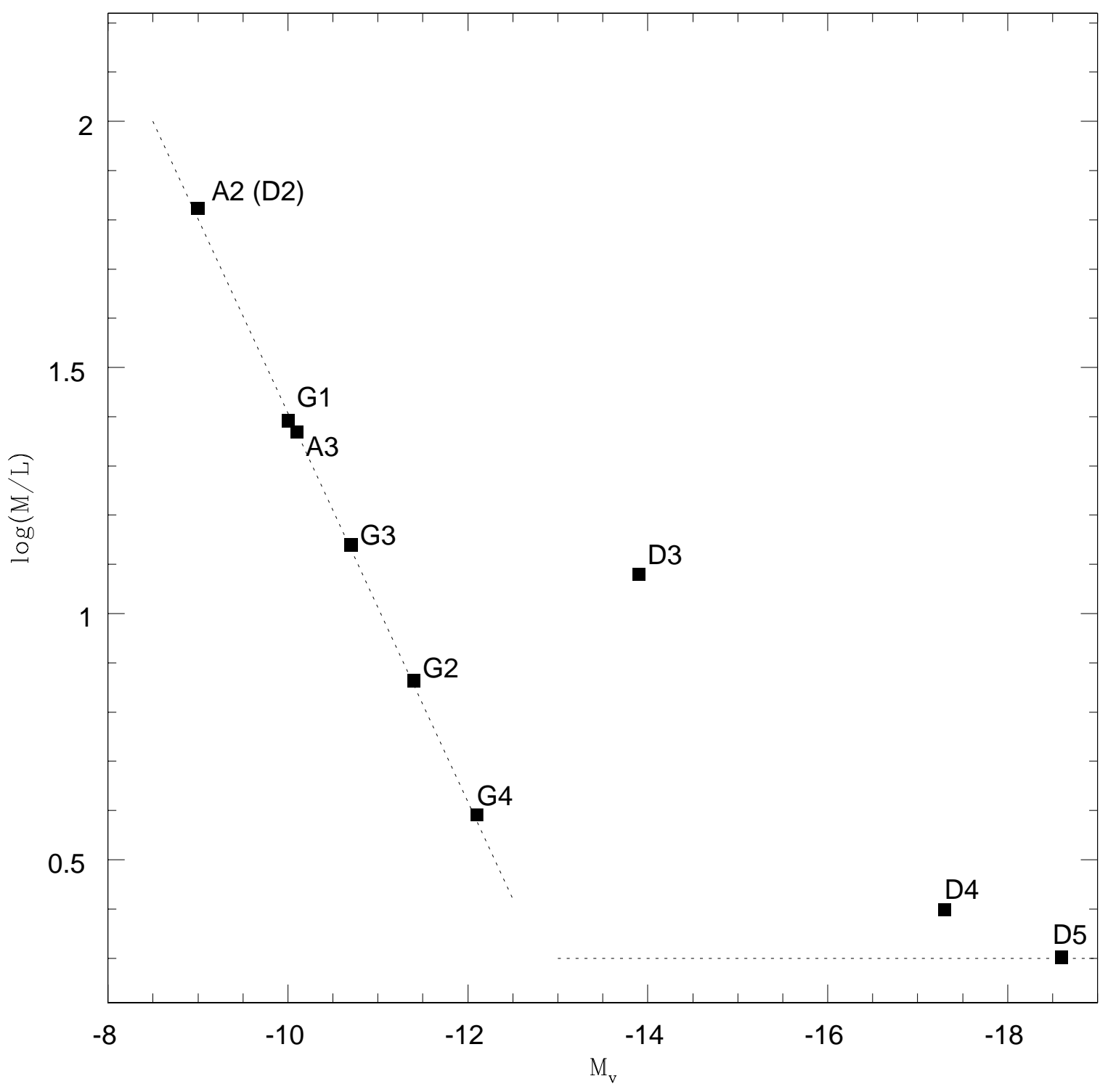

Fig. 13.- Mass-to-light ratio versus $M_{v}$ for models A1-3, D1-5 and G0-5. 